\title{
1 The evolution of the phenylpropanoid pathway entailed pronounced radiations and 2 divergences of enzyme families
}

4 Sophie de Vries $^{1,2^{*}}$, Janine MR Fürst-Jansen ${ }^{2 *}$, Iker Irisarri ${ }^{2,8^{*}}$, Amra Dhabalia Ashok ${ }^{2}$, Till 5 Ischebeck $^{3,4,5}$, Kirstin Feussner ${ }^{3,4}$, Ilka N Abreu ${ }^{3}$, Maike Petersen ${ }^{6}$, Ivo Feussner ${ }^{3,4,5}$, Jan de 6 Vries ${ }^{2,7,8 \#}$

7 1-Heinrich-Heine University Düsseldorf, Population Genetics, Universitätsstr. 1, 40225 Düsseldorf, Germany

82 - University of Goettingen, Institute for Microbiology and Genetics, Department of Applied Bioinformatics,

9 Goldschmidtstr. 1, 37077 Goettingen, Germany

103 -University of Goettingen, Albrecht-von-Haller-Institute for Plant Sciences, Department of Plant Biochemistry, Justus-

11 von-Liebig Weg 11, 37077 Goettingen, Germany

124 - University of Goettingen, Goettingen Center for Molecular Biosciences (GZMB), Goettingen Metabolomics and

13 Lipidomics Laboratory, Justus-von-Liebig Weg 11, 37077 Goettingen, Germany

145 - University of Goettingen, Goettingen Center for Molecular Biosciences (GZMB), Department of Plant Biochemistry,

15 Justus-von-Liebig Weg 11, 37077 Goettingen, Germany

166 -Philipps-Universität Marburg, Institut für Pharmazeutische Biologie und Biotechnologie, Robert-Koch-Str. 4, 35037,

17 Marburg, Germany

187 -University of Goettingen, Goettingen Center for Molecular Biosciences (GZMB), Department of Applied

19 Bioinformatics, Goldschmidtsr. 1, 37077 Goettingen, Germany

208 -University of Goettingen, Campus Institute Data Science (CIDAS), Goldschmidstr. 1, 37077 Goettingen, Germany

*these authors contributed equally

\# author for correspondence:

Jan de Vries

Georg-August University Göttingen, Institute for Microbiology and Genetics, Department of Applied Bioinformatics, Goldschmidtstr. 1, 37077 Göttingen, Germany, phone: +49-551-39-23755

devries.jan@uni-goettingen.de

ORCIDs: Sophie de Vries 0000-0002-5267-8935, Janine MR Fürst-Jansen 0000-0002-5269-8725, Iker Irisarri 0000-00023628-1137, Amra Dhabalia Ashok 0000-0001-5787-6941, Till Ischebeck 0000-0003-0737-3822, Ilka N Abreu 0000-00034728-0161, Kirstin Feussner 0000-0003-1634-8258, Maike Petersen 0000-0001-7769-8556, Ivo Feussner 0000-0002-9888-

AUTHOR EMAILS: sophie.devries@uni-goettingen.de; Janine.fuerst-jansen@uni-goettingen.de; iker.irisarriaedo@unigoettingen.de; amradhabalia.ashok@uni-goettingen.de; $\quad$ tischeb@uni-goettingen.de; $\quad$ kfeussn@uni-goettingen.de; ilkanacif.deabreu@uni-goettingen.de; petersen@mailer.uni-marburg.de; ifeussn@uni-goettingen.de; devries.jan@unigoettingen.de

KEYWORDS: phenylpropanoid biosynthesis; plant evolution; evolution of gene families; evo-physio 


\section{ABSTRACT}

43 Land plants constantly respond to fluctuations in their environment. Part of their response is

44 the production of a diverse repertoire of specialized metabolites. One of the foremost sources

45 for metabolites relevant to environmental responses is the phenylpropanoid pathway, which

46 was long thought to be a land plant-specific adaptation shaped by selective forces in the

47 terrestrial habitat. Recent data have however revealed that streptophyte algae, the algal

48 relatives of land plants, have candidates for the genetic toolkit for phenylpropanoid

49 biosynthesis and produce phenylpropanoid-derived metabolites. Using phylogenetic and

50 sequence analyses, we here show that the enzyme families that orchestrate pivotal steps in

51 phenylpropanoid biosynthesis have independently undergone pronounced radiations and

52 divergence in multiple lineages of major groups of land plants; sister to many of these

53 radiated gene families are streptophyte algal candidates for these enzymes. These radiations

54 suggest a high evolutionary versatility in the enzyme families involved in the

55 phenylpropanoid-derived metabolism across embryophytes. We suggest that this versatility

56 likely translates into functional divergence and may explain the key to one of the defining

57 traits of embryophytes: a rich specialized metabolism.

\section{INTRODUCTION}

60 A diverse profile of specialized metabolites is one of the characteristics of land plants

61 (embryophytes). Almost any aspect of the biology of land plants is underpinned by

62 specialized metabolites-be it the phytohormones that are major modulators upstream in

63 various regulatory hierarchies (Scheres and van der Putten, 2017; Berens et al., 2017;

64 Blázquez et al., 2020) or pigments that give land plants their color and attune photochemical

65 properties (Jahns and Holzwarth, 2012).

A key aspect of the biological relevance of most specialized metabolites is their use under challenging environmental conditions. Indeed, the elaboration of their specialized metabolism is considered one of the drivers for the massive radiation of embryophytes on land (Weng 2013). Moreover, a diversity of specialized metabolism likely played a key role during the earliest steps of plants on land-allowing for the production of compounds that protected land plants against the challenges of the terrestrial environment such as drought and increased UV radiation (Rensing, 2017; de Vries and Archibald, 2018; Fürst-Jansen et al., 2020; Jiao et al., 2020). A major pathway giving rise to a variety of specialized metabolites

74 that act in warding off environmental stressors is the biosynthesis of phenylpropanoids

75 (Dixon and Paiva, 1995; Dixon et al., 2002; Vogt, 2010). 
The phenylpropanoid pathway is the source of precursors for thousands of metabolites with multifaceted functions, and accounts for about $40 \%$ of organic carbon on earth (Vogt, 2010). One facet of these functions is that phenylpropanoid-derived compounds act as structural polymers, foremost among which are the different types of lignin (Ralph et al., 2004; Vanholme et al., 2012; Vanholme et al., 2019). Another prominent facet is that these metabolites act as UV-protecting substances. While some of the best-known UV screens are flavonoids, various other compounds stemming from the phenylpropanoid pathway are equally potent UV protectants (Sheahan, 1996; Booij $\square$ James et al., 2000; Sytar et al. 2018; Xue et al., 2020). The list of links between phenylpropanoid-derived compounds and the response to environmental challenges could be continued; in fact, the response to almost any abiotic stressor that plants face in the terrestrial habitat involves the action of phenylpropanoid-derived compounds (for comprehensive reviews see, e.g., Dixon and Paiva, 1995; Vogt, 2010). Furthermore, phenylpropanoid-derived compounds are involved in the defense responses against plant pathogens in many land plant lineages (Danielsson et al. 2011; Ponce de Leon et al., 2012; König et al., 2014; Overdijk et al., 2016; Carella et al., 91 2019).

All embryophytes make use of the enzymatic routes in the phenylpropanoid pathway. For example, the utilization of flavonoids under UV stress appears to be a conserved response across Embryophyta (Wolf et al., 2010; Clayton et al., 2018). However, not all embryophytes produce the same compounds under the same stress conditions-in contrary, the diversity of compounds is immense. Major differences in the biosynthesis of phenylpropanoid-derived compounds occur in distinct lineages of land plants. This includes specialized roles such as the flower coloration determining anthocyanins that attract pollinators (Miller et al., 2011; Sheehan et al., 2012); such a role of anthocyanins is obviously limited to flowering plants and

101 Piatkowski et al. (2020) phylogenetically inferred that orthologs for the entire anthocyanin

102 biosynthesis pathway are already present in the ancestor of seed plants and more than half of 103 the important orthogroups were already present in the most recent common ancestor of all 104 land plants. An important recent insight into the deep evolutionary roots of flavonoid 105 biosynthesis was the discovery of auronidins - a novel class of red flavonoid pigments that 106 are synthesized in the bryophyte Marchantia polymorpha (Berland et al. 2019). Further, for 107 example, Renault et al. (2017a) reported on the enrichment of the Physcomitrium patens 108 (moss) cuticle in phenolic compounds-an enrichment that hinges on the action of a 109 cytochrome P450 enzyme that is orthologous to enzymes that act in lignin biosynthesis; the 
110 production of lignin might trace its evolutionary roots back to an ancient set of enzymes

111 acting in the production of complex, phenol-enriched polymers (Renault et al., 2019).

112 Carella et al. (2019) showed that the liverwort model plant Marchantia polymorpha triggers

113 phenylpropanoid biosynthesis upon attack by the oomycete phytopathogen Phytophthora

114 palmivora. Similar responses towards phytopathogens are known from gymnosperms (Oliva

115 et al., 2015) and angiosperms (Dixon and Paiva, 1995; Bednarek et al., 2005; Kaur et al.,

116 2010; Chezem et al., 2017; Carella et al., 2019). Thus, all land plants use the core framework

117 of the phenylpropanoid pathway to produce-often lineage-specific-variations of

118 phenylpropanoid derivatives that aid in response to biotic and abiotic stressors.

119 The production of the chemical repertoire of land plants is often catalyzed by

120 members of large enzyme-coding gene families (Shockey et al., 2003; Nelson and Werck-

121 Reichhart, 2011; Renault et al., 2017b), and this also seems to be the case for the enzymes

122 involved in phenylpropanoid biosynthesis (Hamberger et al., 2007; Xu et al., 2009; Vogt,

123 2010). It is thus conceivable that various adaptive forces have shaped the families of enzymes

124 that act in the phenylpropanoid pathway, leading to multiple independent cases of sub- and

125 neofunctionalization (see also Rensing, 2010). An inference of the common (minimal) set of

126 enzymes that were present in the last common ancestors (LCA) of (i) streptophytes, (ii) land

127 plants and their closest streptophyte algal relatives, and (iii) land plants can thus shed light on

128 which enzymatic building blocks evolution acted upon to give rise to the elaborate chassis of

129 the phenylpropanoid pathway.

130 The phenylpropanoid pathway has long been considered to be specific to

131 Embryophyta. However, homologs of the genes coding for the enzymes that constitute the

132 embryophytic phenylpropanoid pathway can be found in extant algal relatives of land plants,

133 suggesting that they were already present in a common ancestor shared by streptophyte algae

134 and land plants (de Vries et al., 2017; Renault et al., 2019; Maeda and Fernie, 2021). Since

135 the beginning of 2020, we have genome data from all major lineages of Streptophyta-except

136 Coleochaetophyceae (Szövényi et al., 2021); only using this extended repertoire of species

137 and sequences allows us to pinpoint which subfamilies and/or which ancestral enzyme of

138 multiple subfamilies were present in the aforementioned LCAs. Compounds that, in land

139 plants, emerge from the phenylpropanoid pathway are indeed found in algae; these include

140 flavonoids and lignin-like compounds in streptophyte algae (Delwiche et al., 1989; Sørensen

141 et al., 2011; Jiao et al., 2020) and core phenylpropanoid building blocks as well as flavonoids

142 in a phylodiverse set of algae (Goiris et al. 2014). Interestingly, lignin-like compounds were

143 even found in distantly-related red macroalgae (Martone et al., 2009)—although this is likely 
144 a case of convergence that builds on an unknown enzymatic framework. However, even

145 within the green lineage (Chloroplastida), the question of the deep evolutionary roots of the 146 phenylpropanoid pathway is wide open.

147 Investigations of the algal relatives of land plants have strongly benefitted from recent 148 progress in phylogenomics on plants and algae. A major outcome of these recent 149 phylogenomic analyses was that the Zygnematophyceae have been pinpointed as the class of 150 algae most closely related to land plants (Wodniok et al., 2011; Wickett et al., 2014; Leebens151 Mack et al., 2019). Hand in hand with these phylogenomic efforts went the generation of 152 genomic (Hori et al., 2014; Nishiyama et al., 2018; Cheng et al., 2019; Wang et al., 2020; 153 Jiao et al., 2020) and transcriptomic data on streptophyte algae (Ju et al., 2015; Rippin et al., 154 2017; de Vries et al., 2018; de Vries et al., 2020). Additionally, critical gaps in the land plant 155 tree of life have been filled; this includes recent publications of the first genomes of 156 liverworts (Bowman et al., 2017), ferns (Li et al., 2018) and hornworts (Szövenyi et al., 2015; 157 Li et al., 2020; Zhang et al., 2020). These data allow for the fine-grained tracing of the 158 evolution of key plant enzyme families across the green tree of life. Recent studies have 159 illuminated the diversity of enzymes in the routes towards flavonoids and anthocyanins as 160 well as the PAL-dependent pathway of salicylic acid biosynthesis via benzoic acid 161 (Piatkowski et al., 2020; Güngör et al., 2021, de Vries et al., 2021).

162 In this study, we infer the evolutionary history of eleven critical enzyme families 163 known to be woven into the mesh of routes from phenylpropanoids to lignin biosynthesis in 164 land plants; we have paid particular attention to the routes leading to the biosynthesis of 165 lignin. We use the new diversity of genomic and transcriptomic data from land plants as well 166 as streptophyte and chlorophyte algae to infer the origin of these large gene families. The 167 datasets were chosen in a manner that they cover the breadth of streptophyte diversity while 168 providing a balanced sampling; the latter is especially relevant in light of the high number of 169 genomes of flowering plants. We aimed to include at least one representative of each of the 170 major lineages of streptophytes in the datasets we surveyed. Our data pinpoint deep homologs 171 of candidate enzymes in streptophyte algae for L-phenylalanine ammonia-lyase (PAL), 4172 coumarate-CoA ligase (4CL), caffeoyl-CoA $\boldsymbol{O}$-methyltransferase (CCoAOMT); further, for 173 streptophyte and chlorophyte algae, we pinpoint homologs for cinnamoyl-CoA reductase 174 (CCR), cinnamyl alcohol dehydrogenase (CAD), and potentially relevant monoacylglycerol 175 lipases (MAGLs). Further, we find that often the functionally characterized enzymes of the 176 core phenylpropanoid and lignin biosynthesis routes derive from lineage-specific radiations, 177 limiting the inference of function outside the model system. Nonetheless we could infer 
178 which subfamilies were present in LCAs along the trajectory of streptophyte evolution, even

179 though ancestral functional inference was limited. That said, for enzyme families with deep

180 homologs, we approximated the function through domain prediction and the conservation (or

181 lack thereof) of key residues of known functional importance. We found that all enzyme

182 families underwent several lineage-specific expansions and losses as well as bursts in growth

183 of enzyme families that occurred early during the radiation of land plants. We hypothesize

184 that lineage-specific expansions in these enzyme families is linked with the diversity of

185 lineage-specific phenylpropanoid derivatives and functions that occur in the species analyzed 186 here.

RESULTS AND DISCUSSION

189 The checkered occurrence of phenylalanine ammonia-lyase among streptophyte algae

190 The conversion of the aromatic amino acid phenylalanine and/or tyrosine into cinnamate 191 and/or $p$-coumarate is the first step of the plant phenylpropanoid pathway (Figure 1). This 192 first committed step is catalyzed by PAL and the bifunctional L-phenylalanine/ L-tyrosine 193 ammonia-lyase (PTAL; Barros and Dixon, 2020). For a long time, it was thought that among 194 Chloroplastida, PAL/PTAL were limited to land plants; their gain was considered to have 195 occurred via a lateral gene transfer event that has occurred at the base of the land plant clade 196 (Emiliani et al., 2009). Recently, however, genes coding for putative PAL-like enzymes were detected in streptophyte algae, such as the filamentous streptophyte alga Klebsormidium nitens (de Vries et al., 2017). In light of the recent surge in available genomes from across the green tree of life, we set out to explore the evolutionary history of PAL.

Using AtPAL1 as a bait sequence, we screened protein data from diverse land plants and all streptophyte algal genomes available. Hits among streptophyte algae fell into three categories: (i) proteins of between 480 (Klebormidium nitens PAL, kf100104_0290_v1.1) and 527 amino acids (two homologs in Chara braunii; g57646_t1 and g34530_t1); (ii) short proteins such as ME1156409C09523 of Mesotaenium endlicherianum, which is 184 amino acid in length; (iii) long proteins of between 991 (Chlorykbus atmophyticus Chrsp482S06115) and 1115 amino acids (Klebsormidium nitens kfl00024_0250_v1.1).

207 Proteins falling into the third category are fusions of an aromatic amino acid lyase domain 208 and a putative tRNA synthetase; homologs of such also occur in land plants (e.g., 209 AT3G02760 and Os05g0150900). The short proteins of category '(ii)' are found in some 210 Zygnematophyceae and are proteins of unknown function with a putative HAL domain. 211 Genomic artefacts leading to this result can be excluded given that these types of protein- 
212 encoding genes have been recovered for independent Zygnematophyceae that are likely >500

213 million years divergent from one another. The proteins in category '(i)' are those with the 214 highest identity to bona fide land plant PALs. This category includes the promising PAL 215 candidate kf100104_0290_v1.1 (see de Vries et al., 2017). We therefore set out to further 216 explore these PAL-like candidates, which noteworthily were only found in the genome of 217 Klebsormidium nitens and Chara braunii and no other streptophyte algal genome data.

218 To understand the evolutionary history of PAL in streptophytes, we computed a maximum likelihood phylogeny (Figure 2). The phylogenetic analysis included the aforementioned PAL homologs from diverse Streptophyta as well as bacterial and fungal PALs. In agreement with previous studies (Emiliani et al., 2009; de Vries et al., 2017), the fungal and bacterial PAL sequences are closely related (bacterial clade: bootstrap support 100; fungal clade: bootstrap support 85) to the clade of land plant PALs (bootstrap support: 96). Additionally, we included diverse eukaryotic and prokaryotic histidine ammonia lyases (HALs) based on the set obtained from de Vries et al. (2017), which, as in this latter study, form two clades with eukaryotic and prokaryotic HALs (bootstrap support 83 and 97). All putative PAL-like candidate sequences from Chara braunii clustered with HAL sequences, one (Chara braunii g66119_t1) with a cyanobacterium (bootstrap support 100), and two (Chara braunii g34530_t1 and Chara braunii g57646_t1) sister to an entire bacterial HAL clade (bootstrap support 100). Both sequences retrieved for Klebsormidium nitens, in contrast, clustered with the PAL clades, one K. nitens kf100024_0250_v1.1 with low support 232 (boostrap level 61) and a rather long branch as sister to plant and fungal PALs, showing that 233 its placement is not fully resolved and that further analyses are required to identify its true 234 identity. The second Klebsormidium nitens sequence, kfl00104_0290_v1.1, clustered within 235 the clade of bacterial PALs (bootstrap support 100), of which some were already functionally 236 characterized-for example, the characterized PAL of Nostoc punctiforme (Moffit et al., 237 2007). This is in agreement with the placement of this protein sequence in de Vries et al. 2382017 and supports it as a putative PAL sequence.

239 In sum, the evolutionary origin of streptophyte PAL appears to be complex and 240 remains obscure: it may be that PAL had a distinct origin in streptophyte algae and land 241 plants, yet the pattern may also be explained by an origin via an endosymbiotic gene transfer 242 from the cyanobacterial plastid progenitor that was retained by streptophytes with a gain of 243 an extra C-terminal domain later in the evolution of embryophytes, resulting in the two 244 distinct PAL clades. It is however important to note that the 3' region of the genomic locus 245 that codes for the shorter $K$. nitens protein kfl00104_0290_v1.1 contains sequence 
246 information that resembles code for the missing C-terminal stretch; thus, the C-terminal

247 stretch might have simply been secondarily lost in K. nitens. Independently, the presence of

248 PALs in fungi further complicates the evolutionary scenario. Rampant gene losses during

249 eukaryotic evolution or convergent domain acquisitions, as well as horizontal gene transfer

250 from plants (as hypothesized by Emiliani et al., 2009) are other scenarios that can explain the

251 evolutionary origin of PALs in streptophytes and thus ultimately in land plants.

Streptophyte algae have an expanded and divergent repertoire of cytochrome $\mathbf{P 4 5 0}$ monooxygenases with no clear $\mathrm{C} 4 \mathrm{H}$ orthologs

After the synthesis of cinnamate by PAL, two routes open up (Figure 1). One of them is the conversion of cinnamate into $p$-coumarate, which is catalyzed by cinnamate 4-hydroxylase (C4H). C4H belongs to the large class of CYP450 enzymes, which are present among all domains of life (Omura, 1999). Among the CYP450 enzymes C4H belongs to the CYP450 subfamily 73 (CYP73). In land plants, CYP450s have undergone massive duplication and subfunctionalization, underpinning the specialized metabolic capabilities of embryophytes (Nelson and Werck-Reichhart, 2011); for example, the CYP73 subfamily belongs to the larger CYP71 clan. The specific CYP450 monoxygenases that fall into the group of $\mathrm{C} 4 \mathrm{H}$ appear to be limited to land plants: clear orthologs can be found in bryophytes and tracheophytes (Emiliani et al., 2009; de Vries et al., 2017). That said, the product of the reaction carried out by $\mathrm{C} 4 \mathrm{H}$ in land plants ( $p$-coumarate) has been detected via UHPLCMS/MS in phylodiverse algae (Goiris et al., 2014). Therefore, there appears to exist a route towards $p$-coumarate that is either independent of $\mathrm{C} 4 \mathrm{H}$ via direct transformation of tyrosine by PTAL or carried out by a highly divergent $\mathrm{C} 4 \mathrm{H}$ homolog. PTALs have so far however been observed in monocots (Barros et al., 2016; Barros and Dixon, 2020), suggesting a different CYP73 subfamily enzyme that may carry out the reaction. Owing to the recent increase in genomic data available for streptophyte algae, we revisited the question of when C4H-based $p$-coumarate might have emerged and explored CYP450 evolution.

We sampled $\mathrm{C} 4 \mathrm{H}$ homologs from seven land plant genomes that had a BLAST bit score (a normalized alignment score) of at least 200, as well as seven streptophyte algal and

275 five chlorophyte algal genomes that had a bit score of at least 100. We aligned all $\mathrm{C} 4 \mathrm{H}$ 276 homologs and computed a maximum likelihood phylogeny (Figure S1). The well277 characterized $\mathrm{C} 4 \mathrm{H}$ of Arabidopsis fell into a clade with full (100 out of 100) bootstrap 278 support; this clade included at least one $\mathrm{C} 4 \mathrm{H}$ homolog from each of the other six land plants 279 genomes, corroborating the notion that all land plants have $\mathrm{C} 4 \mathrm{H}$ orthologs, which appear 
280 conserved in their function from bryophytes to tracheophytes (Russel and Conn, 1967; Urban

281 et al., 1994; Ro et al., 2001; Wohl and Petersen, 2020) and thus since the LCA of land plants.

282 However, no algal sequences fell into this clade. That said, we observed four well-supported 283 clades of streptophyte algal CYP450 enzymes (Figure S1). Investigating the genetic 284 distances, we find that some of the streptophyte algal sequences have a closer genetic 285 distance to the $\mathrm{C} 4 \mathrm{H}$-like clade than to sequences from land plants (including Arabidopsis 286 thaliana) from other CYP450 subfamilies (Table S1). While these sequences remain of 287 unknown function, they are candidates for the CYP450 enzyme family that catalyzes the 288 C4H-function in algae.

\section{A deep split of streptophyte 4CL/ACS}

291 The second route that opens up after the PAL-dependent step is the conversion of cinnamate 292 into cinnamoyl-CoA. This is carried out by the AMP-forming synthetase/ligase 4CL and 293 potentially other enzymes annotated as acyl-CoA synthetases (ACS/ACoS) (Shockey et al., 294 2003; Figure 1). Altogether, these enzymes belong to a large family of distantly related acyl295 activating enzymes (AAEs), such as the long-chain acyl-CoA synthetases (LACS) and many more (Shockey et al., 2003; Figure S2). Homologs with affinity to 4CL appear to occur across chlorophytes and streptophytes (Labeeuw et al., 2015). At least in Arabidopsis, the family of 4CLs has expanded and includes four canonical ("4CL") and nine additional 4CLlike (“4CLL") members, falling into AAE clade IV and V as defined by Shockey et al. (2003). We thus set out to understand what the 4CL repertoire of the last common ancestor of land plants and the one shared with algae might have looked like.

In order to trace the radiation of $4 \mathrm{CLs}$ across the green tree of life, we sampled 4CL homologs from genomes of nine land plants, seven streptophyte algae (plus four transcriptomes of streptophyte algae) and five chlorophyte algae that had a minimum of 400 and a maximum of 1150 amino acids in length and showed affinity to the 4CL clade in a larger phylogenetic survey (Figure S2). We recovered a large clade (bootstrap support 85) that included all bona fide 4CL paralogs and ACOS5 of Arabidopsis thaliana (Figure 3); ACOS5 has been previously associated with the bona fide 4CL clade (Shockey et al., 2003) but it did show only inconsistent activity on typical substrates of 4CL (Costa et al., 2005) and appears to have a very specific function in sporopollenin biosynthesis of pollen (de Azevedo

312 amino acids at sites that bind hydroxycinnamate in typical 4CLs (Figure 3), which might 313 suggest a different natural substrate for ACOS given that affinity is mostly determined by the 
314 binding pocket size (Hu et al. 2010). We further recovered the angiosperm-specific separation 315 defined by Ehlting et al. (1999) into class I and class II 4CLs. Additional lineage-specific 316 radiations occurred, for example in Physcomitrium patens, which fell into a clade of 317 bryophyte sequences (bootstrap support of 70) and in Selaginella moellendorffii (spread out 318 over the fully-supported clade of 4CLs). The common ancestor of land plants appears to have 319 possessed an ACOS5-like and one 4CL-like gene, all other 4CL paralogs in this clade likely 320 emerged later during land plant evolution. Clustering with AAE clade IV (including 321 AtACOS5, and At4CL1,2,3 and 5 (bootstrap support 85)) are sequences from five 322 streptophyte algae. Each of the five streptophyte algae possesses one homolog to these five 323 types of AMP-forming ligases with 4-coumarate-CoA synthesizing activity. When we 324 predicted the tertiary structure of Chlorokybus atmophyticus Chrsp175S02417 and Penium margaritaceum 006213.t1 via Iterative Threading ASSEmbly Refinement (I-TASSER; Zhang 326 2008), we recovered, in both cases, firefly luciferases as best match (TM-scores 0.852 and 327 0.918; 1BA3 and 2D1S; Franks et al., 1998; Nakatsu et al., 2006). Investigating the putative 328 structure of the other streptophyte algal sequences (Zygnema circumcarinatum DN42558_c0_g1_i1, Spirogyra pratensis 3442_c2_g1_i6, and Klebsormidium nitens 330 00016_0470_v1.1), however, always recovered Populus tomentosa 4CL (3A9U; Hu et al., 3312010 ) as their closest structural analog (TM scores of 0.957, 0.969, and 0.961, respectively).

332 Hence, we hypothesize that a 4CL/ACOS5-like encoding gene was present in the last 333 common ancestor of all streptophytes. Underpinning this hypothesis is that the sequence of 334 the amino acids in the binding pocket in the streptophyte algal 4CL homologs is consistent 335 with that of 4CL homologs from other land plants (including that of Arabidopsis thaliana; 336 Figure 3). Further, the amino acids relevant for the enzymatic function (i.e., the residues 337 KQK involved in adenylation, nucleophilic substitution and coumaroyl-AMP cleavage) are 338 also conserved across most 4CL/ACOS5 sequences, including those of the streptophyte 339 algae. Variation in these residues is already apparent in 4CLL homologs and outside of the 340 4CL/ACOS5/4CLL clade these residues show high variability (Figure 3).

341 A similar pattern was observed when we investigated the domain structure of all 342 recovered sequences. Most of the 4CL/ACOS5-like sequences contained four domains: 343 Phosphopantetheine binding ACP domain (IPR025110), AMP-binding, conserved site 344 (IPR020845), AMP-dependent synthetase-like superfamily (IPR042099), AMP-dependent 345 synthetase/ligase (IPR000873; Figure S3). There were four exceptions to this pattern. They 346 include one sequence from the water fern Azolla filiculoides (Azfis0013.g013344) and two 347 hornwort sequences from Anthoceros agrestis BONN (Sc2ySwM344.2803.3 and 
348 Sc2ySwM344.2803.4), which all missed the Phosphopantetheine binding ACP domain

349 (IPR025110). The other exception was AtACOS5, which is the only sequence in this clade 350 that missed the conserved AMP-binding site (IPR020845). The domain pattern is similar 351 across the 4CLL-like clade, too. Yet, more sequences miss either the IPR025110 and/or the 352 IPR020845 domain. The streptophyte algal sequences within the ACOS5/4CL clade 353 contained all four domains, whereas algal sequences outside of this clade missed at least 354 one-but recovered several other domains. These additional domains are not conserved 355 within the phylogenetic subclades of these algal sequences and only exceptionally occur in 356 the 4CL/ACOS4/4CLL (two sequences) or Other AMP-dependent synthetase and ligase 357 family protein clades (two sequences).

358 We recovered a second clade of spermatophyte sequences (bootstrap support 89) 359 representing AAE clade V enzymes, which contains 4CLL8 and several other ATP-ligases of 360 Arabidopsis thaliana with predicted 4CL activity, including OPCL1 (matching 4CL-like 5 361 with a $100 \%$ amino acid identity according to Uniprot). Sequences in this clade, however, 362 diverge in the amino acids that are involved in the formation of the binding pocket in the 363 canonical 4CLs (Figure 3), which might point to a different substrate preference of the 364 enzymes in this clade. In fact, OPCL1 and many of these "4CLLs" (e.g., AT5G63380, 365 AT1G20500, AT4G05160) showed higher activity on fatty acids and fatty acid-derived 366 precursors for the phytohormone jasmonic acid than cinnamate-derived compounds in an in vitro substrate survey carried out by Kienow et al. (2008). It is thus questionable that the enzymes of this clade act as bona fide 4CLs. Homologs to these sequences are found in

369 Brachypodium distachyon and Picea abies, suggesting an origin in the last common ancestor 370 of seed plants followed by two duplication events with either (a) both taking place in the last 371 common ancestor of angiosperms or (b) one early on in the LCA of seed plants and the 372 second in the LCA of angiosperms. Bootstrap support to include the Picea abies sequences in 373 the clade containing AT5G63380 (4CLL9) is however low (bootstrap 54). Each duplication 374 event was followed by independent lineage-specific radiations giving rise to a whole plethora 375 of possible candidates for $4 \mathrm{CL}$, but also a large evolutionary potential with regard to substrate 376 specificity and flexibility. The 4CLL clade of spermatophytes is nested within a larger, lowly 377 supported clade (bootstrap 65) that included sequences from across the land plant tree of life. 378 Here, pronounced and independent expansion occurred in most of the major lineages of land 379 plants, leading to large clades of, for example, proteins of the hornwort Anthoceros and the 380 lycophyte Selaginella. As noted above, most of the Selaginella and some of the Anthoceros 381 homologs retained the conserved KQK residues required for the catalytic activity but others 
382 did not (Figure 3), which suggests the presence of species-specific functions. Outside of the 383 entire 4CL-ACOS and 4CLL clade ('Streptophyte 4CL/ACOS/4CLL-likes'; bootstrap 84)

384 clustered various highly divergent ATP-dependent synthetases and ligases that exist 385 throughout the green tree of life including sequences from chlorophytes. None of these 386 synthetases and ligases retained the catalytic triad KQK.

387 Altogether, our phylogenetic data indicated that a 4CL/ACOS5-like encoding gene 388 was present at the base of Streptophyta. Domain annotation and the analysis of amino acid 389 patterns in the binding pocket and functional sites support this idea. Further, the similarity of 390 these residues between the candidates of streptophyte algal homologs for 4CLs and the 391 sequences of 4CL proteins with high activity on cinnamate derivatives as substrates (see also 392 Costa et al., 2005), indicates that 4CL activity may evolved more than 700 million years ago 393 in streptophytes.

Patchy distribution of CCR-likes in streptophyte algae and massive independent radiations in land plants

En route to the production of different lignin monomers is the NADPH-dependent reduction of the activated acyl-group of the phenylpropanoid backbone molecules. This first step towards an aldehyde functionality is carried out by CCR (Figure 1), which falls into a larger family of NADPH-dependent reductases, including dihydroflavonol reductases (DFRs) and DFR-likes (DFL) (Lacombe et al., 1997; Devic et al., 1999). We previously reported the presence of CCR-like protein sequences in streptophyte algae (de Vries et al. 2017). Since these previous analyses, however, genome data on additional major lineages of land plants and streptophyte algae have become available.

With these new data at hand, covering most major lineages of streptophyte algae and all major lineages of land plants, it is now possible to infer the evolutionary history of CCRlike and DFR-like sequences. We computed a maximum likelihood phylogeny of CCR homologs with a minimum of 220 amino acids that we detected in genomes of 15 land plants, seven streptophyte algae, and five chlorophytes; additionally, we included sequences found in

410 the transcriptomes of the Zygnematophyceae Spirogyra pratensis (de Vries et al., 2020), 411 Zygnema circumcarinatum (de Vries et al., 2018), and the Coleochaetophyceae Coleochaete 412 orbicularis (Ju et al., 2015; Figure 4).

413 The CCR homologs were distributed over several major clades. This included the 414 CCRL/DRL-like sequences described as TETRAKETIDE $\alpha$-PYRONE REDUCTASE 415 (TKPR) by Grienenberger et al. (2010), which is an important enzyme acting in the 
416 production of sporopollenin. We recovered a well-supported clade of TKPR1 homologs 417 (bootstrap support of 84) and fully supported clade of TKPR2 homologs. Both clades of 418 TKPRs contained homologs from across the diversity of land plants, bolstering the idea that 419 TKPR1 and TKPR2 split early during plant evolution (Grienenberger et al., 2010)_before 420 the most recent common ancestor of land plants came about. Our domain structure analyses 421 found that TKPR1 possessed the NAD-dependent epimerase/dehydratase (IPR001509) and 422 NAD(P)-binding domain superfamily (IPR036291) domains, which appear to be present in 423 most sequences included in the phylogeny as well as the Tetraketide alpha-pyrone reductase 4241 (IPR033267) domain (Figure S4). In contrast, TKPR2 only encoded the first two domains, 425 which is more similar to what is found in the CCR clade.

426 The bona fide CCRs and CCR-likes were spread out over two clades. These two 427 clades were nested in a weakly-supported monophylum (bootstrap support of 53), which was 428 sub-divided into four medium to fully supported clades. A fully supported clade of CCR-likes 429 (including AT4G30470 and AT2G23910) included sequences from across embryophytes; we 430 coined this monophylum CRL-A. AtCCRL1 and AtCCRL2 appear to be co-orthologs to one 431 sequence in the Brassicaceae Capsella grandiflora (0380s0077.1.p), thus our data suggest a 432 limited distribution of direct orthologs to CCRL1 and CCRL2. Yet the two sequences 433 together fall into a large clade, here coined CRL-B (bootstrap support 73), that contained 434 sequences from all major lineages of tracheophytes. Another medium-supported clade 435 (bootstrap support 73) included the bona fide CCRs, CCR1 and CCR2, of Arabidopsis. The 436 duplication that resulted in these two CCRs occurred earliest in the common ancestor of all 437 rosids and latest in the common ancestor of Brassicaceae, yet the CCR clade included 438 homologs from across tracheophytes. Many of these lineages appear to have expanded their 439 own repertoire from one CCR1/2 homolog that was present in the last common ancestor of 440 tracheophytes. Interestingly, a clade of divergent monocot CCRs display several 441 replacements in key amino acids involved in the binding of the substrate's phenolic ring, in 442 particular a replacement of non-polar aliphatic Ile to aromatic Tyr/Phe (Figure S5), which 443 might reflect a difference in substrate affinity. The catalytic triad SYK (Figure S5; Pan et al. 444 2014) is required for enzymatic activity and is overall conserved across CCR/DFR-likes and 445 FLDHs. Interestingly, several CRL-As possess non-conservative amino acid replacements 446 from large phenolic (Tyr/Phe) to smaller (His, Leu, Ser, Gly) amino acids, which might 447 suggest divergent substrate affinities for CRL-As. This is consistent with the domain 448 structure of many CRL-A sequences, which often lack the NAD-dependent 449 epimerase/dehydratase (IPR001509) domain, but possess additional domains such as 3- $\beta$ - 
450 hydroxysteroid dehydrogenase/isomerase (IPR002225) or match an additional NAD(P)-

451 binding domain (IPR016040; Figure S4). This pattern is only occasionally occurring in 452 sequences from the CCR or other CRL clades.

453 Finally, there is a third clade with a bootstrap support of 69 that included tracheophyte 454 sequences (forming a sub-clade with a bootstrap value of 90) and a single Marchantia 455 polymorpha homolog; we coined this clade CRL-C. Altogether, this suggests that the LCA of 456 all land plants had two homologs of CCRs/CRLs: one CRL-A and one CRL-B, CRL-C or 457 CCR homolog. In vascular plants, duplications have resulted in sub-clades of the CRL458 B/CRL-C/CCR homologs.

459 Within the larger clade that encases the DFRs, DFRLs, CCRs and CCRLs 460 ("Chloroplastida CCR/DFR-likes"; bootstrap 86), one supported clade of Zygnematophyceae 461 (bootstrap support 77) and one supported clade of chlorophyte and streptophyte algae 462 (bootstrap support 76) exists. This points to a distinct DFR/DFRL/CCR/CCRL clade that 463 arose in the ancestor of Zygnematophyceae, yet its placement within the phylogeny other 464 than it belonging to the larger DFR/DFRL/CCR/CCRL clade is uncertain. The divergent 465 pattern of amino acids, which perform substrate and cofactor binding in land plants, suggest 466 that these algal homologs might vary in the substrate and enzymatic activity compared to 467 plant CCR/DFRs (Figure S5) It is, however, certain that within land plants, a pronounced 468 radiation of CCRs occurred.

\section{CADs are present across the green lineage}

471 The second reduction step of the activated acyl-group of the phenylpropanoid backbone and 472 one of the last steps in lignin biosynthesis is the production of phenylpropanoid-derived 473 alcohols from the corresponding aldehydes. An example is the conversion of $p$-coumaroyl 474 aldehyde into $p$-coumaryl alcohol (Kim et al., 2004; Pan et al., 2014). The required reduction 475 is catalyzed by CAD (Figure 1), which is the rate determining enzyme by which lignin is 476 produced (Gross et al., 1973; Mansell et al., 1974). In Arabidopsis thaliana, there are at least 477 eleven enzymes belonging to the CAD family. Enzymes of the CAD family have been 478 divided into five major groups, of which group IV was described as monocot-specific 479 (Saballos et al., 2009). In our previous studies many of the chlorophyte and streptophyte 480 potential CAD homologs, identified mostly from transcriptomes and few genomes of algae, 481 were described as CAD-like or CAD group II/III-affiliated (de Vries et al. 2017; de Vries et 482 al. 2020). Sequences clustering with those of CAD group II have been characterized as 483 sinapyl alcohol dehydrogenase (SAD) or show predicted structural similarity to SAD 
484 enzymes (Guo et al. 2010; de Vries et al. 2017). Additionally, some SADs appear involved 485 not in the synthesis of lignin but defense compounds such as lignans (Suzuki and Umezawa, 486 2007; Guo et al., 2010; Saleem et al., 2010; Barakate et al., 2011), and it may thus be that 487 CAD group II is functionally versatile.

488 Here, we used the 11 canonical CAD sequences to understand the diversity in CAD 489 homologs across streptophytes. This includes also CAD group II sequences, for which 490 homologs in other species may have other substrate specificities and thus are involved in 491 different steps of the phenylpropanoid pathway (Barakat et al., 2009; Guo et al., 2010). We 492 computed a phylogeny of CAD homologs (Figure 5) detected in phylodiverse Chloroplastida. 493 While the resolution of the backbone is weak, we recovered all five CAD groups defined by 494 Saballos et al. (2009). All the CAD groups were resolved as land plant-specific clades of 495 CAD homologs with robust support. Each clade contained a varying set of major land plant 496 lineages (described below); the clades of putative streptophyte algal CAD homologs 497 contained both fewer proteins and fall in-between the five CAD-groups. Hence, this more 498 phylodiverse dataset tells a more complicated evolutionary history for CAD homologs than 499 the less-diverse data from de Vries et al. (2017).

$500 \quad$ Our data suggest that the common ancestor of Zygnematophyceae and land plants 501 may have possessed two CAD-like genes, which was followed by lineage-specific radiations. 502 While it appears - based on the overall topology of the tree-appealing to suggest that one 503 gene gave rise to CAD-group $\mathrm{V}$ and the other ancestral gene was the basis of CAD-group I to 504 IV, the low statistical support for the backbone of the phylogeny does not allow to confirm 505 such hypothesis (Figure 5). We can infer that the earliest land plants likely inherited a few (or 506 just one) CAD homolog from their algal progenitors. Most of the radiation of CADs has 507 occurred in plants dwelling on land. Of the canonical CAD group-and based on those part 508 of the topology with good bootstrap support-CAD-group V (containing AtCAD1) is the 509 only group present in all major land plant lineages (Figure 5). CAD-group I was likely 510 present in the LCA of tracheophytes, as it includes sequences from angiosperms, 511 gymnosperms, ferns, and the lycophyte Selaginella moellendorffii. CAD-groups II, III, and 512 IV include only angiosperm sequences_-but note that with very weak bootstrap support (53) 513 a sequence from the gymnosperm Gnetum montanum associates with the group $514 \mathrm{CADII} / \mathrm{III} / \mathrm{IV}$; likely, expansion resulted in the ancestral gene of CAD-group II and III, which 515 diverged into CAD group III genes in angiosperms and after another expansion CAD-group 516 II originated in the LCA of dicots. This is in contrast to de Vries et al. (2017), where the 517 streptophyte algal CAD-like sequences were clustering with CAD group II/III sequences, but 
518 resembles the placement of transcriptomic CAD-like sequences from Spirogyra pratensis and

519 Mougotia sp. in an already more diverse phylogenetic analysis (de Vries et al., 2020). This is

520 a clear case where including a larger diversity of streptophyte sequences to the analysis

521 enables us to better understand the complexity of the evolution of highly radiated gene

522 families. An analysis of the residues salient to CAD function showed a general conservation

523 of residues involved in zinc and $\mathrm{NADP}^{+}$binding across Chloroplastida CAD-like sequences

524 (cf. Youn et al. 2006), whereas the residues in the binding pocket are generally less conserved

525 in these sequences (Figure 5). However, within a canonical CAD-groups or a CAD-like clade

526 we see a general conservation of residues in the binding pocket. The binding pockets of each

527 CAD-like clade appear different than those of the canonical CAD-groups. Domain analyses

528 suggest that some of the CAD-like sequences of streptophyte algae do not encode all of the

529 five domains present in most canonical CAD sequences, yet many of the

530 Zygnematophyceaen CAD-like sequences encode all of these five domains. Similar patterns

531 emerge for other CAD-like sequences (Figure S6).

532 All CAD groups are shaped by multiple lineage-specific duplications and losses. This

533 hampers the inference of function and substrate specificity of the diverse CAD-like

534 sequences. Additionally, several lineages have originated a variety of CAD homologs that are

535 not yet designated to previous groups. In the absence of functional data, we however will not

536 give them a group designation but rather designate them as lineage-specific CAD-homologs

537 of unknown SAD or CAD function. CAD-like homologs found in streptophyte algae show

538 similar functional residues to other CAD-likes of land plants (not included in any of the five

539 clades of canonical CADs), including at the binding pockets-the pattern of residues is

540 similar to what is observed for other land plant sequences in-between the bona fide CAD

541 groups. The bona fide CAD groups showed a more homogeneous pattern of functional

542 residues.

543 Overall, both the topology of the phylogenetic tree and the conservation of key

544 residues point to (i) a deep evolutionary origin of CAD homologs and (ii) independent

545 radiations of CADs - not only in land plants but also in streptophyte algae.

547 Massive independent radiations of acyltransferases and scattered candidates in 548 streptophyte algae

549 A versatile group of enzymes that are important for the processes leading up to the lignins but 550 also compounds with antioxidant and antimicrobial properties are the BAHD acyltransferases 551 (named after the first enzymes characterized for this family BEAT, AHCT, HCBT, and DAT; 
552 see also D’Auria, 2006). Most prominent among these are the versatile hydroxycinnamoyl-

553 CoA shikimate/quinate hydroxycinnamoyltransferases (HCT) (Eudes et al., 2016). Recently,

554 Kriegshauser et al. (2020) reported on the functional conservation of the HCT homologs

555 found in bryophytes with those of seed plants.

556 We computed a phylogeny including phylodiverse acyltransferases (Figure S7).

557 Overall, the topology of the tree corroborates the findings of Kriegshauser et al. (2020) that a

558 clear clade of HCT proteins first emerged in land plants—streptophyte algal sequences were

559 few, divergent from HCT, and scattered over the tree without clear affinity to characterized

560 acyltransferases. Without functional analyses, there is no solid foundation for predicting their

561 function-making them exciting candidates for future studies. What our data, however,

562 clearly reveal is that the last common ancestor of land plants likely had an expanded

563 repertoire of acyltransferases that further diversified during the radiation of plants on land.

\section{A clear clade of C3Hs is limited to land plants}

566 At several steps of the phenylpropanoid pathway, enzymes belonging to the cytochrome P450

567 family CYP98A, within the large CYP71 clan (Nelson and Werck-Reichhart, 2011), carry out

568 hydroxylations of $p$-coumarate-derived compounds (such as $p$-coumaroyl esters; for function

569 of $\mathrm{C} 3 \mathrm{H}$ see Figure 1). In land plants, these hydroxylations are important for the production of

570 lignins, lignans, volatile phenylpropanoids, coumarins, and many more phenylpropanoidderived compounds. Previously, de Vries et al. (2017) described the detection of C3H in all

572 land plants and one putative $\mathrm{C} 3 \mathrm{H}$ ortholog in Klebsormidium nitens. Now, with genomic gaps

573 in the streptophyte tree of life filled, we revisited the distribution of $\mathrm{C} 3 \mathrm{H}$.

574 The number $\mathrm{C} 3 \mathrm{H}$ homologs detected in genomes of 15 land plants, 7 streptophyte 575 algae, and five chlorophytes and in the transcriptomes of Spirogyra pratensis (de Vries et al., 576 2020), Zygnema circumcarinatum (de Vries et al., 2018), and Coleochaete orbicularis (Ju et 577 al., 2015) varied strongly between lineages. When sampling the sequences via BLAST (with 578 AT2G40890 as query sequence), we thus included either (a) all sequences that had a bit score 579 of at least 100 , or (b) the top five hits. We aligned all sequences, cropped them to the 580 alignable region and computed a maximum likelihood phylogeny (Figure 6).

581 A clade of CYP98A included sequences from all major lineages of land plants. This 582 suggests that at least one CYP98A sequence was present in the LCA of all land plants. Based 583 on the lineages included here, it appears that from this single copy gene, radiations occurred 584 in the dicot lineages and Amborella trichocarpa. A single copy remained in the bryophytes, 585 lycophytes, ferns, gymnosperms, and monocots. The clade of CYP98A8 and CYP98A9 was 
586 in our dataset limited to the Brassicaceae Arabidopsis thaliana and Capsella grandiflora 587 (bootstrap value 100). These two enzymes function in a route derived from the 588 phenylpropanoid pathway and are involved in the formation of $N^{1}, N^{5}$-di(hydroxyferuloyl)$589 N^{10}$-sinapoylspermidine (Matsuno et al., 2009). The CYP98A8/9 clade falls into the larger 590 CYP98A clade (bootstrap support 100) together with the $\mathrm{C} 3 \mathrm{H}$ sequences, suggesting that 591 they are the closest paralogs of $\mathrm{C} 3 \mathrm{H}$ in Arabidopsis thaliana and Capsella grandiflora. 592 Analyses of their substrate recognition sites (SRS; cf. Rupasinghe et al. 2003) support the 593 divergent functional roles between CYP98A8/9 and canonical C3H. In particular, the first 594 two SRS (SRS1 and SRS2) show various distinct amino acid differences between the 595 CYP98A8/9 clade and the C3H clade (Figure S8). SRS1 and 2 are predicted to be involved in binding of the substrate tails, which exhibit strong variation in size (Rupasinghe et al. 2003), and thus may be critical for the substrate specificity of these paralogs. Indeed, SRS1 and 2 are

598 the two SRSs showing the strongest variation across the entire phylogeny, including also 599 other CYP450 subfamilies (Figure 6), corroborating this hypothesis.

600 We recovered additional land plant-specific clades of CYP450 enzymes (Figure 6) 601 such as one containing TRANPARENT TESTA7 (TT7)-like sequences (bootstrap support 602 92); TT7 is a cytochrome P450 75B enzyme that is involved in flavonoid biosynthesis 603 (Tanaka et al., 1997; Schoenbohm et al., 2000). The BLAST search used for sampling C3H 604 homologs further recovered AtCYP71B and AtCYP76C members. In our phylogenetic 605 analysis, we inferred that AtCYP71B34 and AtCYP71B35 were likely born out of an 606 Arabidopsis-specific duplication, while CYP71B enzymes in general are present across 607 angiosperms. In contrast, $A t C Y P 76 C 1$ and $A t C Y P 76 C 4$ appear to have originated prior to the 608 split of Arabidopsis thaliana and Capsella grandiflora; a CYP76C4 ortholog appears to have 609 been lost in the latter plant species. The CYP76C clade may also be represented in other 610 species outside of angiosperms, because we find a sequence from Gnetum montanum 611 clustering with these sequences with a bootstrap support of 87 . The rather long branch 612 warrants attention and would require a more CYP76C focused phylogenetic analysis, which 613 is not the point of this paper.

614 The algal sequences showed strong divergence to the $\mathrm{C} 3 \mathrm{Hs}$, forming only a larger 615 (fully supported) streptophyte-specific clade with all the recovered and functionally diverse 616 CYP450 enzymes (Figure 6). Domain structures of these streptophyte algal sequences is the 617 same to what is observed for the $\mathrm{C} 3 \mathrm{H}$ homologs, but is in general conserved across the 618 phylogeny independent of the CYP450 subfamily assignment (Figure S9). Only few scattered 619 exceptions occur. Given the low support of most of the tree backbone, the role(s) of the 
620 streptophyte algal homologs detected here remains elusive. Most of the streptophyte algae

621 show independent radiations of their CYP450 enzymes complicating functional predictions

622 even further. Thus, while there are interesting CYP450 candidates in streptophyte algae, a

623 clear $\mathrm{C} 3 \mathrm{H}$ clade likely first arose early during the evolution of embryophytes.

625 Monoacylglycerol lipases: multiple early radiations, independent subfunctionalization, 626 and the origin of CSE

627 The conversion of caffeoyl-5-O-shikimate to caffeic acid may be a step along the 628 biosynthetic routes that lead to the production of G- and S-lignins in certain vascular plants 629 (Figure 1). The enzyme responsible for this step is caffeoyl-5- $O$-shikimate esterase (CSE).

630 CSE converts caffeoyl-5- $O$-shikimate to caffeic acid and was hypothesized to act together 631 with 4CL/ACOS5 to circumvent the catalysis of caffeoyl-5- $O$-shikimate to caffeoyl-CoA via 632 HCT (Vanholme et al. 2013). The latter pathway was proposed for tobacco by Hoffmann et 633 al. (2003) and confirmed for Arabidopsis thaliana in vitro by Vanholme and colleagues 634 (2013). Yet, based on cse mutants in Arabidopsis thaliana, they suggested that synthesis of 635 caffeoyl-CoA is more likely to occur via CSE and 4CL/ACOS5 than directly from caffeoyl 636 shikimate by HCT in planta. That said, in the model grasses Brachypodium distachyon and 637 Zea mays no CSE orthologs are present and crude extracts from these species show little 638 signs for the characteristic esterase activity (Ha et al., 2016). On the other hand, non-vascular 639 plants such as the model system Physcomitrium patens possess homologs of these enzymes 640 (Renault et al., 2017a), which suggests a secondary loss of CSE in the respective monocots. 641 CSE belongs to the family of putative monoacylglycerol lipases (MAGL). MAGLs are found 642 across eukaryotes and functional analyses in human, yeast and Arabidopsis have shown that 643 they possess monoacylglycerol lipase activity (Labar et al., 2010; Aschauer et al., 2016; Kim 644 et al., 2016). In contrast to other MAGLs of Arabidopsis thaliana, AtCSE (MAGL3) was 645 found to exhibit no hydrolytic activity on monoacylglycerols (MAGs) as a substrate (Kim et 646 al., 2016) — which applies to other enzymes of Arabidopsis thaliana that belong to the family 647 of MAGLs, too. Indeed, out of the 16 MAGLs that Kim and colleagues (2016) tested, only 648 MAGL6 and 8 showed high activity on MAG as substrate. Given the functional diversity in 649 MAGLs (Kim et al. 2016) and the unequal distribution of caffeoyl-5- $O$-shikimate across 650 embryophytes, functional analyses are required to fully understand how easily MAGLs can 651 lose or gain their MAGL activity. Yet, phylogenetic analyses can pinpoint the diversity of the 652 family across the green lineage. 
Here we use phylogenetic analysis to pinpoint the distributions of the diverse MAGL

654 families, including CSE across the green lineage. In total, we recovered all 16 MAGL 655 sequences of Arabidopsis thaliana in the similarity search; using maximum likelihood 656 phylogenetics, we recovered clades for all the 16 MAGLs; some MAGL clades are widely 657 distributed throughout streptophytes, while others appear to have originated in embryophytes, 658 where they have again undergone lineage-specific expansions. Essentially, we recovered two 659 large clades: one restricted to streptophytes, containing homologs of MAGL2, 4 and 13; the 660 other has representation in chlorophytes as well and includes homologs of MAGL1, 3, 5, 6, 7, $8,9,10,11,12,14,15$ and 16 (Figure 7).

Focusing on the MAGL2/4/13 clade first, we observe that MAGL13 has representation in angiosperms, gymnosperms and ferns, suggesting its origin to be in the LCA of tracheophytes, while MAGL2 and 4 came from a duplication event before the split between Arabidopsis and Capsella. However, MAGL2/4 orthologs are present in other species including Picea abies, suggesting that the common ancestor of seed plants possessed a MAGL2/4-like and a MAGL13 gene. Forming a clade with MAGL2/4/13 are lycophyte, bryophyte and streptophyte algal sequences, which in general branch in an order expected based on their species phylogeny (although within species duplication events have occurred). This suggests that already at the base of streptophytes a MAGL2/4/13-like gene was present.

In the second large clade that includes also chlorophyte sequences, we find the clade containing the CSE/MAGL3 orthologs. This clade includes sequences from both vascular and non-vascular plants, pointing to an origin of CSE in the last common ancestor of land plants.

674 This adds support for a secondary loss in those monocots without a CSE ortholog. Despite the 675 origin of CSE in the common ancestor of land plants and a clear CSE ortholog in 676 Physcomitrium patens (3c19_14430V3.1.p), the substrate of CSE, caffeoyl-5-O-shikimate, 677 was not detected in crude extracts of the moss (Renault et al., 2017a). The HCT-based reaction leading to caffeoyl-CoA has been confirmed in vitro using moss HCT (Kriegshauser et al. 2021). Hence, the CSE homologs of Physcomitrium patens may have another function. Indeed, the atypical function of $A t \mathrm{CSE}$, together with the lack of other MAGL family members, to act on MAGs (Kim et al. 2016), suggests that the functional spectrum of the MAGL family is not very limited in land plants.

683 Members of the MAGL family share several conserved motifs across diverse 684 eukaryotes. One such motif (amino acid positions 132-141 in MAGL6 and 167-176 in 685 CSE/MAGL3) is situated in a region likely involved in substrate binding based on the crystal 686 structure of human MAGL (Labar et al., 2010). Within this motif a leucine in position 
687 number four is found in a diverse set of 249 mammal and Sauria MAGLs investigated here 688 (Figure 7, inset) and most plant MAGLS including MAGL6 and 8. This is followed by 689 another hydrophobic amino acid (I in mammals/Sauria and V or L in most plant MAGLs). It 690 is striking that exactly these highly conserved amino acids are changed to a phenylalanine 691 and a serine in the Arabidopsis CSE and some homologs from other species. These changes 692 from two very hydrophobic amino acids to an aromatic and a hydrophilic one could be one of 693 the reasons for a change in substrate specificity from a substrate with a hydrophobic acyl 694 chain to a more hydrophilic substrate with aromatic properties. Based on this hypothesis, 695 CSEs would be restricted to some of the members of this clade that, however, stem from across the diversity of land plants.

MAGL1 appears to have originated prior to the split of angio- and gymnosperms, while specific MAGL14 and 16 orthologs likely arose after the split of asterids and rosids, but a MAGL14/16 ortholog was likely present in the LCA of land plants. MAGL15, like MAGL1, originated prior to the split of gymno- and angiosperms, and MAGL5 appeared to come from a duplication of MAGL15 later on possibly in the ancestors of dicots. Interestingly, a MAGL5/15-like sequence was already encoded in the genome of the ancestor of streptophytes. The same is true for a MAGL6/7/8/9/10/11/12-like gene, which similar to the MAGL5/15-like genes shows independent paths of radiation in streptophyte algae and land plants. MAGL6,7,8,10 and 11 are only present in the here included Brassicaceae, while a MAGL6/7/8/10/11-like gene was already present in the common ancestor of spermatophytes. The same evolutionary history describes the scenario under which MAGL9 and 12 originated.

Finding the MAGL6/7/8/10/11 subclade specifically expanded in seed plants is noteworthy. At least AtMAGL8 localizes to lipid droplets (Kim et al., 2016), which are structures found in various photosynthetic eukaryotes but are well-known from seeds. Thus, expansion of this clade might be a read-out of spermatophyte-specific additions to the ancient set of proteins relevant to LD formation and function (see de Vries and Ischebeck, 2020).

All in all, the MAGLs have experienced an early radiation in streptophytes. Given that even the Arabidopsis MAGLs without detectable activity on MAG (see Kim et al., 2016) do not form one single monophylum, it is conceivable that subfunctionalization of members of the MAGL family occurred multiple times independently. This may likewise be true for all

719 here. The versatility in functional evolution of MAGLs makes it difficult to make robust 720 predictions of putative MAGL functions. 


\section{COMT: convergence and complexity}

723 In angiosperms, ferulate 5-hydroxylase $(\mathrm{F} 5 \mathrm{H})$ and caffeate $O$-methyltransferase (COMT) 724 carry out important catalytic steps along the route from $p$-coumaroyl-CoA to S-lignin. COMT 725 catalyzes the methylation of caffeic acid or 5-hydroxyferulic acid, the product formed by 726 F5H. Like C3H and C4H, F5H belongs to the large CYP450 clan 71 (Nelson and Werck727 Reichhart, 2011). The function of F5H evolved at least twice in P450 enzymes, once in the 728 ancestor of angiosperms and once in the ancestor of lycophytes (Weng et al., 2008; Weng and 729 Chapple, 2010).

$730 \quad$ For angiosperm F5H, no clear putative orthologs were found outside of flowering 731 plants and likewise no clear orthologs were found for the lycophyte F5H (i.e. "SmF5H"), 732 which forms a separate clade from the angiosperm F5H sequences (Figure S10). This 733 corroborates previous results (de Vries et al., 2017) and is in agreement with the hypothesis 734 that F5H function evolved at least twice in the evolution of land plants (Weng et al., 2008). 735 Additionally, the average pairwise identity of the $\mathrm{F} 5 \mathrm{H}$ homologs was quite low (18.5\%) 736 hampering robust phylogenetic analyses. We thus did not further delve into the evolution of 737 F5H. COMT however caught our attention.

738 The lycophyte Selaginella moellendorffii not only uses a genetically distant F5H 739 enzyme; the same appears to be true for COMT (Weng et al., 2011). This highlights a 740 promiscuity for substrate specificity and activity in P450 enzymes that is yet to be discovered 741 and mere orthology analyses can only go so far as to discover putative candidates. Using 742 phylogenetics, we explored the diversity of methyltransferases by screening for sequences 743 homologous to COMT/OMT1 of Arabidopsis thaliana across our phylodiverse dataset. This 744 approach identified not only clear orthologs but can also serve as a backbone to map relevant 745 mutations facilitating in functionally convergence in this group of enzymes and by that may highlight possible candidates for in vivo and in vitro studies.

For most clades of land plant methyltransferases, based on the here recovered

748 topology, predicting a putative function was not straightforward. This applied even more so 749 to the homologs of COMT/OMT found in chlorophyte and streptophyte algae. We recovered 750 a clade of methyltransferases that included chlorophyte and streptophyte algae as well as 751 diverse land plant sequences (coined 'Chloroplastida OMT' in Figure 8); among these 752 clustered Arabidopsis thaliana proteins such as COMT, indole glucosinolate 753 methyltransferases (IGMT), and nicotinate $N$-methyltransferase (NANMT; see Li et al., 754 2017) — hence different methyltransferases that act on a range of aromatic compounds. What 
755 this means for the presence of a putative COMT in algae is obscure. However, it corroborates 756 the previously observed patchy detection of OMT1 across the green lineage based on a 757 reciprocal BLASTp searches (de Vries et al., 2017). Clear orthologs of AtCOMT were only 758 detected for a few angiosperms, notably not including any monocot sequence that we 759 included in our dataset. Of all methyltransferases in our dataset, only NANMT formed a 760 clade of clear orthologs that included more than one major lineage of land plants by encasing 761 sequences from angiosperms and Picea abies (bootstrap support 87). All other orthogroups appear, like COMT, to be restricted to only a few of the included angiosperm lineages.

The lycophyte Selaginella moellendorffii has a COMT that is distantly related to OMT of angiosperms. It appears to have acquired its OMT activity through convergent evolution and was coined SmCOMT (Weng et al., 2011). In agreement with this, SmCOMT did not cluster with the AtOMT1 sequence in our analyses. Instead, it forms its own (weak) clade with only one other sequence from Selaginella moellendorffii (bootstrap support 63). The other SmCOMT-like sequences (described in Weng et al., 2011) were distributed over the phylogeny and appear to be specific to Selaginella moellendorffii. Nonetheless, this pattern highlights a certain versatility in the evolutionary history of substrate specificity of $O$ methyltransferases in land plants. This appears to be only logical, noting the large lineagespecific expansions in the larger clade of $O$-methyltransferases that encompasses all $O$ methyltransferases from Arabidopsis thaliana-that is COMT, IGMTs, NANMT and $\mathrm{N}$ acetylserotonin $O$-methyltransferase (ASMT; for more on this enzyme see, e.g., Tan et al., 2012; Byeon et al., 2016) (bootstrap-support 99). Within this clade fall also algal sequences from chlorophytes and streptophyte algae. Their position within the clade is undetermined due to low bootstrap support. These sequences appear highly divergent, many of them cluster with rather long branches. Yet, some of the sequences from our previous analysis found a reciprocal BLASTp hit to AtCOMT, including Klebesormidium nitens 00158_0100v1.1 that clusters in a fully supported clade of Klebesormidium paralogs; these sequences are promising candidates to explore caffeic acid $O$-methyltransferase activity. Indeed, when we modelled the tertiary structure of Klebesormidium nitens 00158_0100v1.1 and Mesotaenium endlicherianum ME000591S08520 using I-TASSER (Zhang, 2008), we recovered Medicago sativa and Lolium perenne COMT as its closest structural analogs (1KYZ; Zubieta et al., 2002; 3P9C; Louie et al., 2010; TM-scores 0.865 and 0.956, respectively). independent radiations in this large gene family. Given the observed convergent evolution of 
789 COMT activity in Selaginella moellendorffii, the question of whether there is COMT activity across Streptophyta remains wide open.

To gain a first insight into whether COMT activity can be expected from other streptophyte lineages, we investigated the conservation of residues relevant for the function of COMT, including those that form the substrate binding pocket (Figure 8). The functional residues were identified from COMT of Lolium perenne (Louie et al., 2010). Across our phylogeny, these residues differ between the clades of canonical ASMT, NANMT, COMT and IGMT, while they are conserved within them (Figure 8). The binding pocket of AtOMT1 and its orthologs consist of the amino acid pattern MSNGGG, whereas the pattern for the residues important for the function of the enzyme is HDE. While HDE appears conserved across the majority of sequences analyzed here, independent of the specific function of the enzyme (e.g. ASMT, IGMTs and COMT all have the pattern HDE), the binding pocket is highly variable among the functionally characterized enzymes. This suggests that the reaction-determining residues are those that form the binding pocket and not those that are catalytically important. This seems logical given that all these enzymes catalyze similar types of reactions. The triple $\mathrm{G}$ in the binding pocket is also far more conserved across the entire phylogeny, with only few exceptions occurring, while the first three residues are highly variable. Indeed, the COMT-specific MSN motif is not present in the functionally characterized COMT from Selaginella moellendorfii, rather it is MTN, which however is a change between similar amino acids (Ser to Thr). Apart from the COMT orthologs and SmCOMT sequence no other sequences from any other lineage encode the binding pocket pattern $\mathrm{M}(\mathrm{S} / \mathrm{T}) \mathrm{NGGG}$, suggesting that none has a canonical preference for binding 5hydroxyconiferalaldehyde. Yet, several homologs_-including those of streptophyte algae-

812 would have the ability to catalyze the reaction based on the conservation of the residue 813 pattern HDE. What is more, all the sequences that could not be properly identified as 814 orthologs to ASMT, NANMT, COMT and IGMT (with the exception of SmCOMT) show no

815 similarity in their first three residues of the binding pocket to either of these enzyme families.

816 This would suggest that the enzymes from most streptophyte lineages included in this 817 analysis use different substrates than those functionally characterized in Arabidopsis 818 thaliana.

\section{CCoAOMT-like sequences emerged in Phragmoplastophyta}

821 Within the phenylpropanoid pathway, caffeoyl-CoA $O$-methyltransferase (CCoAOMT) and 822 most of its homologs are the enzymes that catalyze the first committed step to many of at 
823 least two types of lignin (S- and G-lignin). These enzymes methylate caffeoyl-CoA and thus

824 give rise to feruloyl-CoA (Ye et al., 1994; Ye and Varner, 1995; Martz et al., 1998; Do et al.,

825 2007; Vanholme et al., 2012); in the past, it was also proposed that after the conversion to 5'-

826 hydroxy-feruloyl-CoA, CCoAOMT can methylate this compound to produce Sinapoyl-CoA

827 (Maury et al., 1999; Ferrer et al., 2005). One of the paralogs that exist in Arabidopsis

828 thaliana (tapetum-specific $O$-methyltransferase [TSM1], AT1G67990) however, shows

829 activity towards a coniferyl derivative that is formed at $N^{10}$ by F5H starting from $N^{1}, N^{5}, N^{10}$ -

830 tris-(hydroxyferuloyl) spermidine (Fellenberg et al., 2009); TSM1 catalyzes the production of

$831 N^{1}, N^{5}$-bis-(hydroyferuloyl)- $N^{10}$-synapoylspermidine (Fellenberg et al., 2009). Thus,

832 CCoAOMTs appear to be versatile in their substrate specificity and can act on different steps

833 in the phenylpropanoid pathway.

834 Here we used phylogenetics to disentangle the distribution of these enzymes across

835 the green lineage. A duplication gave rise to the genes encoding the functionally divergent

836 enzymes AtCCoAMT and AtTSM1 (bootstrap support 77; Figure 9). These two

837 methyltransferases are embedded in a larger clade containing the other CCoAOMT enzymes

838 AtCCoAOMT1, AtCCoAOMT7 and AtCCoAOMT-like (AT1G24735). The latter appears to

839 be specific to the Brassicaceae included in this dataset, while homologs of AtCCoAOMT7

840 occur across dicots and were detected in Amborella trichopoda, but were absent from the

841 included monocots. Only AtCCoAOMT1 had a wider distribution. Its cluster (bootstrap

842 support 79) contains angiosperms, gymnosperms, lycophytes, ferns and bryophytes,

843 excluding the sequenced hornworts from the genus Anthoceros (Figure 9). Assuming a

844 monophyly of Bryophyta (Puttick et al., 2018), this suggests a loss of CCoAOMT1 in at least

845 the sequenced Anthoceros species, and that CCOAOMT1 was present in the common ancestor

846 of land plants. Lineage-specific duplications of CCOAOMT1 appear to have happened,

847 indicated by the expansions seen in tobacco, spruce, the lycophyte Selaginella moellendorffii

848 and the water fern Azolla filiculoides. The expanded repertoire of sequences in monocots and

849 Gnetum indicate additional lineage-specific duplications outside of the CCoAOMT clade.

850 The case of TSM1 suggests that neo-functionalization can easily occur within this type of 851 methyltransferases. We noted that the residues involved in substrate binding (Ferrer et al.

852 2005) are identical in AtCCoAMT and AtTSM1 (Figure 9). A possible explanation might be

853 that the make-up of binding pocket allows for a certain versatility in substrates. Given these

854 observations, the paralogs within this and the other CCOAOMT clades cannot be assumed to

855 hold the function of CCoAOMTs. Likewise, it cannot be ruled out that their LCA may have

856 had this function. As sister to the methyltransferase clade, including the CCoAOMT 
857 homologs lies a cluster of genes encoding putative candidates for streptophyte algal 858 CCoAOMTs. These were limited to representatives of the two streptophyte algal lineages 859 closest to land plants: the Coleochateophyceae Coleochaete scutata and Coleochaete 860 orbicularis as well as the Zygnematophyceae Spirogloea muscicola. These algal sequences 861 have the same domain structure as the majority of all $S$-adenosyl- $L$-methionine (SAM)862 dependent methyltransferases included in the phylogeny (Figure S11). Only single sequences, 863 scattered across the phylogeny and diversity of species included here, vary in their domain 864 structure showing a loss of a domain loss, a gain of an additional domain, or both. The 865 analyses of the specific functional residues gave more insights into the streptophyte algal 866 sequences within the clade of SAM-dependent methyltransferases in the peripheral routes of the phenylpropanoid pathway. These algal sequences maintain the residues involved in ion and cofactor binding, but differ strongly in the substrate binding site (cf. Ferrer et al. 2005; 869 Figure 9).

870 Altogether, it appears that the family of CCoAOMT-like proteins has its origin in 871 Phragmoplastophyta. Clarifying the function of the putative CCoAOMT-like enzymes in 872 Coleochateophyceae and Zygnematophyceae has the potential to shed light on a 873 synapomorphy with physiological relevance.

\section{Conclusion}

876 All genes for enzymes that act in early steps in the chassis of the phenylpropanoid pathway 877 investigated here (Figure 1) can be traced back to the LCA of land plants with the exception 878 of COMT (Figure 10); most of these can even be traced back to some ancestor that land 879 plants shared with streptophyte algae. While most of our knowledge on how these genes 880 work comes from angiosperms, this does not capture the sequence diversity in enzymes_and 881 it underpins the versatility in producing specialized metabolites.

882 Our data pinpoint that most of the enzymes have undergone massive lineage-specific 883 expansions. A lineage-specific expansion is palpable even despite the fact that sampling of sequences across the Streptophyta is still strongly biased towards seed plants. These data offer a framework for pinpointing those candidate genes/enzymes that are bound to shed light on the evolution of key enzymatic steps-and novel ones. Such work is exemplified by studies on Selaginella or bryophyte model systems such as Physcomitrium patens.

888 Characterizing enzymes that are even more divergent from what we know from angiosperms 889 should yield surprising insights and novel routes in this bountiful pathway. 


\section{MATERIAL AND METHODS}

\section{Dataset of protein sequences and screening for homologs}

893 We downloaded protein data from: (a) genomes of fifteen land plants: Anthoceros agrestis as

894 well as Anthoceros punctatus (Li et al., 2020), Amborella trichopoda (Amborella Genome 895 Project, 2013), Arabidopsis thaliana (Lamesch et al., 2010), Azolla filiculoides (Li et al., 896 2018), Brachypodium distachyon (The International Brachypodium Initiative, 2010), 897 Capsella grandiflora (Slotte et al., 2013), Gnetum montanum (Wan et al., 2018), Marchantia 898 polymorpha (Bowman et al., 2017), Nicotiana tabacum (Sierro et al., 2014), Oryza sativa 899 (Ouyang et al., 2007), Picea abies (Nystedt et al., 2013), Physcomitrium patens (Lang et al., 900 2018), Salvinia cucullata (Li et al., 2018), Selaginella moellendorffii (Banks et al., 2011), and 901 Theobroma cacao (Argout et al., 2011); (b) the genomes of seven streptophyte algae: 902 Chlorokybus atmophyticus (Wang et al., 2020), Chara braunii (Nishiyama et al., 2018), 903 Klebsormidium nitens (Hori et al., 2014), Mesotaenium endlicherianum (Cheng et al., 2019), 904 Mesostigma viride (Wang et al., 2020), Penium margaritaceum (Jiao et al., 2020), Spirogloea 905 muscicola (Cheng et al., 2019); (c) the genomes of five chlorophytes: Bathycoccus prasinos 906 (Moreau et al., 2012), Chlamydomonas reinhardtii (Merchant et al., 2007), Coccomyxa 907 subellipsoidea (Blanc et al., 2012), Micromonas pusilla, Micromonas sp. (Worden et al., 908 2009), Ostreococcus lucimarinus (Palenik et al., 2007), Ulva mutabilis (De Clerck et al., 909 2018), Volvox carteri (Prochnik et al., 2010). Additionally, we included sequences found in 910 the transcriptomes of Spirogyra pratensis (de Vries et al., 2020), Zygnema circumcarinatum 911 (de Vries et al., 2018), and Coleochaete orbicularis (Ju et al., 2015).

912 For each of the protein families we investigated here, the representative Arabidopsis 913 thaliana protein was used as a query sequence for a BLASTp against this dataset. Initially, 914 we considered all homologs recovered at a cutoff level of $10^{-7}$. However, due to the large size

915 of the protein families (i.e. high number of well-supported homologs obtained), refinement of 916 the datasets was carried out as described in the individual sections for these enzymes in the 917 Results and Discussion section.

918

919 Alignments, phylogenetic analysis, and primary sequence analysis

920 Using the homologs detected based on the above described BLASTp search for a given 921 enzyme, we generated alignments using MAFFT v7.453 (Katoh and Standley, 2013) with a 922 L-INS-I approach. Alignments were cropped, if necessary, to retain conserved domains that 923 were alignable for all homologs; alignments are provided in Supplemental Datasets S1 to 924 S11. We computed maximum likelihood phylogenies using IQ-TREE multicore version 1.5.5 
925 (Nguyen et al., 2015), with 100 bootstrap replicates. To determine the best model, we used 926 ModelFinder (Kalyaanamoorthy et al., 2017) and picked the best models based on the 927 Bayesian Information Criterion. The best models were: LG+G4 (Le and Gascuel, 2008) for 928 4CL, CCR, CCoAOMT; LG+I+G4 for PAL, CAD, MAGL/CSE, COMT, and for the 929 preliminary phylogeny of $4 \mathrm{CL}$; $\mathrm{LG}+\mathrm{F}+\mathrm{I}+\mathrm{G} 4$ for $\mathrm{C} 4 \mathrm{H}, \mathrm{F} 5 \mathrm{H}$, and $\mathrm{C} 3 \mathrm{H}$; WAG+F+G4 (Whelan 930 and Goldman, 2001) for HCT.

931 Protein structure prediction was carried out using the sequences as input in the online 932 Iterative Threading ASSEmbly Refinement (I-TASSER; Zhang, 2008; Yang et al., 2015). 933 Functional residue analyses were based on published structural analyses (Rupasinghe et al., 934 2003; Ferrer et al., 2005, Youn et al., 2006; Hu et al., 2010; Pan et al., 2014) and alignments 935 were viewed with SeaView v.4 (Gouy et al., 2009) and plotted with ETE3 (Huerta-Cepas et 936 al., 2016).

\section{Protein domain predictions}

939 Protein domains for all protein sequences for the enzyme families 4CL, CCR, C3H, CAD and 940 CCOAOMT included in the phylogenies were predicted using InterProScan version 5.47-82.0

941 (Jones et al., 2014). The presence or absence of protein domains were mapped onto the 942 phylogenies of the afore mentioned gene families as presence/absence heatmaps, which were 943 visualized using iTOL v6 (Letunic and Bork, 2019).

\section{ACKNOWLEDGEMENTS}

946 J.M.R.F.-J. is grateful for being supported by the Ph.D. program "Microbiology and 947 Biochemistry" within the framework of the "Göttingen Graduate Center for Neurosciences, 948 Biophysics, and Molecular Biosciences" (GGNB) at the University of Goettingen; A.D.A. is 949 grateful for being supported through the International Max Planck Research School (IMPRS) 950 for Genome Science. J.d.V. thanks the European Research Council for funding under the 951 European Union's Horizon 2020 research and innovation programme (Grant Agreement No. 952 852725; ERC-StG “TerreStriAL”). M.P., I.F., and J.d.V. are grateful for support through the 953 German Research Foundation (DFG) within the framework of the Priority Programme 954 "MAdLand - Molecular Adaptation to Land: Plant Evolution to Change" (SPP 2237; VR 955 132/4-1; PE 360/37-1; FE 446/14-1) 
958 Figure 1. Enzymes involved in the biosynthesis of phenylpropanoid-derived compounds

959 investigated here. A simplified schematic of the phenylpropanoid pathway and its routes to

960 different derivatives are shown. Boxes indicate enzyme families, which are mentioned above

961 each box and color-coded. Their coloration is the same as in Figure 10. Dotted lines indicate

962 putative steps in the pathway.

963

964 Figure 2. A phylogenetic framework for the origin of streptophyte PAL. PAL and HAL

965 homologs were screened for in fifteen land plant, seven streptophyte algae, and five

966 chlorophytes. Among Chloroplastida, PAL homologs were only recovered from genomes of

967 land plants and the streptophyte algae Klebsormidium nitens and Chara braunii. From all

968 detected homologs, a rooted maximum likelihood phylogeny was computed using LG+I+G4

969 as model for protein evolution (chosen according to BIC). 100 bootstrap replicates were

970 computed; only bootstrap values $\geq 50$ are shown and bootstrap values of 100 are depicted by a

971 filled dot. Colored font and dots correspond to the support recovered for the higher-order

972 clades labeled on the right of the phylogenies.

974 Figure 3. 4CL homologs occur across Streptophyta. 4CL homologs were sampled from 975 protein data of nine land plant, seven streptophyte algal and five chlorophyte algal genomes.

976 Only protein sequences with a minimum length of 400 and a maximum length of 1150 amino 977 acids were included. From all detected homologs, a rooted maximum likelihood phylogeny 978 was computed using LG+G4 as model for protein evolution (chosen according to BIC). 100 979 bootstrap replicates were computed; only bootstrap values $\geq 50$ are shown and bootstrap 980 values of 100 are depicted by a filled dot. Colored font and dots correspond to the support 981 recovered for the higher-order clades labeled on the right of the phylogenies. On the right we 982 show key residues for substrate binding and function of canonical 4CL as reported by Hu et 983 al. (2010).

985 Figure 4. The complex evolutionary history of CCR in Chloroplastida. CCR homologs of 986 a minimum of 220 amino acids were sampled from protein data of 15 land plant, seven 987 streptophyte algal, and five chlorophyte algal genomes; additionally, we included sequences 988 found in the transcriptomes of Spirogyra pratensis (de Vries et al., 2020), Zygnema 989 circumcarinatum (de Vries et al., 2018), and Coleochaete orbicularis (Ju et al., 2015). From 990 all detected homologs, an unrooted maximum likelihood phylogeny was computed using $991 \mathrm{LG}+\mathrm{G} 4$ as model for protein evolution (chosen according to BIC). 100 bootstrap replicates 
992 were computed; only bootstrap values $\geq 50$ are shown and bootstrap values of 100 are

993 depicted by a filled dot. Colored font and dots correspond to the support recovered for the

994 higher-order clades labeled on the right of the phylogenies.

Figure 5. Phylogenetic analysis highlights CAD candidates across Chloroplastida. CAD

997 homologs were sampled from protein data from fifteen land plant, seven streptophyte algal,

998 and five chlorophyte algal genomes as well as sequences found in the transcriptomes of

999 Spirogyra pratensis (de Vries et al., 2020), Coleochaete scutata and Zygnema

1000 circumcarinatum (de Vries et al., 2018), and C. orbicularis (Ju et al., 2015). From all

1001 detected homologs, an unrooted maximum likelihood phylogeny was computed using

$1002 \mathrm{LG}+\mathrm{I}+\mathrm{G} 4$ as model for protein evolution (chosen according to BIC). 100 bootstrap replicates

1003 were computed; only bootstrap values $\geq 50$ are shown and bootstrap values of 100 are

1004 depicted by a filled dot. Colored font and dots correspond to the support recovered for the

1005 higher-order clades labeled on the right of the phylogenies. The five groups of CADs were

1006 named in accordance with Saballos et al. (2009). Next to the sequence labels residues from

1007 the binding pocket, $\mathrm{NADP}^{+}$- and $\mathrm{Zn}^{2+}$ binding are shown — based on Youn et al. (2016).

1008

1009 Figure 6. A clade of $\mathbf{C 3 H}$ orthologs originated at the base of land plants. $\mathrm{C} 3 \mathrm{H}$ homologs were sampled from protein data of genomes of fifteen land plants, seven streptophyte algae, and five chlorophytes; additionally, sequences found in the transcriptomes of Spirogyra

1012 pratensis (de Vries et al., 2020), Zygnema circumcarinatum (de Vries et al., 2018), and 1013 Coleochaete orbicularis (Ju et al., 2015) were included. For downstream analyses, we used 1014 either (a) all sequences that had a bit score of at least 100 or (b) the top five hits. We aligned 1015 all sequences, cropped them to the alignable region and computed an unrooted maximum 1016 likelihood phylogeny was computed using $\mathrm{LG}+\mathrm{F}+\mathrm{I}+\mathrm{G} 4$ as model for protein evolution 1017 (chosen according to BIC). 100 bootstrap replicates were computed; only bootstrap values $1018 \geq 50$ are shown and bootstrap values of 100 are depicted by a filled dot. Colored font and dots 1019 correspond to the support recovered for the higher-order clades labeled on the right of the 1020 phylogenies. Two large clades that contained only (a) Anthoceros and (b) chlorophyte and 1021 streptophyte algal sequences were collapsed; the full tree is shown in Figure S12.

1023 Figure 7. The occurrence of MAGLs across diverse Streptophyta and a phylogenetic 1024 framework for the deep evolutionary roots of CSE. MAGL/CSE homologs were sampled 1025 from protein data from 15 land plant, seven streptophyte algal, and five chlorophyte algal 
1026 genomes as well as sequences found in the transcriptomes of Spirogyra pratensis (de Vries et

1027 al., 2020), Coleochaete scutata and Zygnema circumcarinatum (de Vries et al., 2018), and

1028 Coleochaete orbicularis (Ju et al., 2015). From all detected homologs, an unrooted maximum

1029 likelihood phylogeny was computed using $\mathrm{LG}+\mathrm{I}+\mathrm{G} 4$ as model for protein evolution (chosen

1030 according to BIC). 100 bootstrap replicates were computed; only bootstrap values $\geq 50$ are

1031 shown and bootstrap values of 100 are depicted by a filled dot. Colored font and dots

1032 correspond to the support recovered for the higher-order clades labeled on the right of the

1033 phylogenies. Purple font highlights those streptophyte algal sequences that share the

1034 conserved alpha helix cap domain with CSE. Logos are based on a motif (amino acids 132-

1035141 in MAGL6 and 167-176 in CSE/MAGL3) that is situated in a region likely involved in

1036 substrate binding based on the crystal structure of human MAGL.

1038 Figure 8. Low resolution on the complex evolutionary history of COMT. We explored

1039 the diversity of methyltransferases by screening for sequences homologous to Arabidopsis

1040 COMT/OMT1 across genome data from fifteen land plant, seven streptophyte algae, and five

1041 chlorophytes; additionally, we included sequences found in the transcriptomes of Spirogyra

1042 pratensis (de Vries et al., 2020), Zygnema circumcarinatum (de Vries et al., 2018), and

1043 Coleochaete orbicularis (Ju et al., 2015). From all detected homologs, an unrooted maximum

1044 likelihood phylogeny of 226 sequences was computed using LG+I+G4 as model for protein

1045 evolution (chosen according to BIC). 100 bootstrap replicates were computed; only bootstrap

1046 values $\geq 50$ are shown and bootstrap values of 100 are depicted by a filled dot. Colored font

1047 and dots correspond to the support recovered for the higher-order clades labeled on the right

1048 of the phylogenies. Blue font highlights streptophyte algal sequences; bold font pinpoints

1049 those, that recovered land plant COMT as closest structural analogs in I-TASSER-based

1050 modeling. On the right we show residues important for substrate binding and function of

1051 canonical COMT as reported by Louie et al. (2010).

1053 Figure 9. A phylogenetic framework for the evolutionary origin of CCoAOMTs in

1054 Phragmoplastophyta. CCoAOMT homologs were sampled from protein data from sixteen

1055 land plant, seven streptophyte algal, and five chlorophyte algal genomes as well as sequences

1056 found in the transcriptomes of Spirogyra pratensis (de Vries et al., 2020),

1057 Coleochaete scutata and Zygnema circumcarinatum (de Vries et al., 2018), and Coleochaete

1058 orbicularis (Ju et al., 2015). From all detected homologs, an unrooted maximum likelihood

1059 phylogeny of 138 sequences was computed using LG+G4 as model for protein evolution 
1060 (chosen according to BIC). 100 bootstrap replicates were computed; only bootstrap values

$1061 \geq 50$ are shown and bootstrap values of 100 are depicted by a filled dot. Colored font and dots

1062 correspond to the support recovered for the higher-order clades labeled on the right of the

1063 phylogenies. Purple font highlights those streptophyte algal sequences that share the

1064 conserved alpha helix cap domain with CSE. The alignment on the right shows functionally

1065 characterized sites involved in substrate, ion and co-factor recognition of CCoAOMT (Ferrer

1066 et al., 2005).

1067

Figure 10. A summary of the proposed evolutionary trajectory of key enzymes in the

phenylpropanoid pathway across the green lineage. At the bottom is a cladogram of the

green lineage. The most recent common ancestors (MRCA) of Chloroplastida, Streptophyta,

Phragmoplastophyta, Bryophyta, Embryophyta and Tracheophyta are indicated at their

respective nodes. On top the cladogram is the proposed evolutionary trajectory of the enzyme

families PAL, C4H, 4CL, CCR, CAD, C3H, CSE/MAGL (CSE), and CCoAOMT. The names of the enzyme families are indicated on the left of the trajectory. The enzyme (sub)families present in a specific common ancestor have been plotted onto the respective nodes of the cladogram below the evolutionary scenario of the enzyme families involved in the phenylpropanoid pathway and lignin biosynthesis. White dots indicate absence/loss of a gene family one dot indicates the presence of one representative of the gene family and several dots indicate an expansion (two or more members of the gene family) in at least one species of the represented lineages in the cladogram. Colors are chosen to distinguish different enzyme families and subfamilies. Question marks label sequences of ambiguous affiliation.

\section{REFERENCES}

1084 Amborella Genome Project (2013) The Amborella genome and the evolution of flowering plants. Science, 342, 1241089

Argout, X., Salse, J., Aury, J.-M., Guiltinan, M. J., Droc, G., Gouzy, J., ... Lanaud, C. (2011). The genome of Theobroma cacao. Nature Genetics, 43, 101-108. Aschauer, P., Rengachari, S., Lichtenegger, J., Schittmayer, M., Padmanabha Das, K. M., Mayer, N., ... Oberer, M. (2016) Crystal structure of the Saccharomyces cerevisiae monoglyceride lipase Yju3p. Biochimica et Biophysica Acta (BBA) - Molecular and Cell 
1092 Banks, J. A., Nishiyama, T., Hasebe, M., Bowman, J.L., Gribskov, N., dePamhilis, C., Albert, V. A., ... Grigoriev, I. V. (2011). The Selaginella genome identifies genetic changes associated with the evolution of vascular plants. Science, 332, 960-963.

1095 Barakat, A., Bagniewska-Zadworna, A., Choi, A. Plakkat, U., DiLoreto, D. S., Yellanki, P., 1096 \& Carlson, J. E. (2009). The cinnamyl alcohol dehydrogenase gene family in Populus: 1097 phylogeny, organization, and expression. BMC Plant Biology 9, 26.

1098 Barakate, A., Stephens, J., Goldie, A., Hunter, W.N., Marshall, D., Hancock, R.D., Lapierre, C., Morreel, K., Boerjan, W., \& Halpin, C. (2011). Syringyl lignin is unaltered by severe sinapyl alcohol dehydrogenase suppression in tobacco. The Plant Cell, 23, 4492-4506.

Barros, J., Serrani-Yarce, J.C., Chen, F., Baxter, D., Venables, B.J., \& Dixon, R.A. (2016). Role of bifunctional ammonia-lyase in grass cell wall biosynthesis. Nature Plants, 2, 16050.

Berens, M.L., Berry, H.M., Mine, A., Argueso, C.T., \& Tsuda, K. (2017). Evolution of hormone signaling networks in plant defense. Annual Review of Phytopathology, 55,

1113 Berland, H., Albert, N.W., Stavland, A., Jordheim, M., McGhie, T.K., Zhou, Y., Zhang, H.,

Blanc, G., Agarkova, I., Grimwood, J., Kuo, A., Brueggeman, A., Dunigan, D. D., ... Van Etten, J. L. (2012). The genome of the polar eukaryotic microalga Coccomyxa subellipsoidea reveals traits of cold adaptation. Genome Biology, 13, R39.

1121 Blázquez, M.A., Nelson, D.C., \& Weijers, D. (2020). Evolution of plant hormone response 1122 pathways. Annual Review of Plant Biology, 71, 327-353.

1123 Booij-James, I.S., Dube, S.K., Jansen, M.A.K., Edelman, M., \& Mattoo, A.K. (2000). 1124 Ultraviolet-B radiation impacts light-mediated turnover of the photosystem II reaction 
1125

1126

1127

1128

1129

1130

1131

1132

1133

1134

1135

1136

1137

1138

1139

1140

center heterodimer in Arabidopsis mutants altered in phenolic metabolism. Plant Physiology, 124, 1275-1284

Bowman, J. L., Kohchi, T., Yamato, K.T., Jenkins, J., Shu, S., Ishizaki, K., ... Schmutz, J. (2017). Insights into land plant evolution garnered from the Marchantia polymorpha genome. Cell, 171, 287-304.

Byeon, Y., Lee, H. J., Lee, H. Y., \& Back, K. (2016). Cloning and functional characterization of the Arabidopsis $\mathrm{N}$-acetylserotonin $\mathrm{O}$-methyltransferase responsible for melatonin synthesis. Journal of Pineal Research, 60, 65-73.

Carella, P., Gogleva, A., Hoey, D.J., Bridgen, A.J., Stolze, S.C., Nakagami, H., \& Schornack, S. (2019). Conserved biochemical defenses underpin host responses to oomycete infection in an early-divergent land plant lineage. Current Biology, 29, 2282-2294.e5.

Cheng, S., Xian, W., Fu, Y., Marin, B., Keller, J., Wu, T., ... Melkonian, M. (2019) Genomes of subaerial Zygnematophyceae provide insights into land plant evolution. Cell, 179, 1057-1067.e14.

Chezem, W.R., Memon, A., Li, F.-S., Weng, J.-K., Clay, N.K. (2017). SG2-type R2R3-MYB transcription factor MYB15 controls defense-induced lignification and basal immunity in Arabidopsis. The Plant Cell, 29, 1907-1926.

Clayton, W. A., Albert, N. W., Thrimawithana, A. H., McGhie, T. K., Deroles, S. C., Schwinn, K. E., ... Davies, K. M. (2018). UVR8 $\square$ mediated induction of flavonoid biosynthesis for UVB tolerance is conserved between the liverwort Marchantia polymorpha and flowering plants. Plant Journal, 96, 503-517.

Costa, M.A., Bedgar, D.L., Moinuddin, S.G.A., Kim, K.-W., Cardenas, C.L., Cochrane, F.C., ... Lewis, N. G. (2005). Characterization in vitro and in vivo of the putative multigene 4coumarate:CoA ligase network in Arabidopsis: syringyl lignin and sinapate/sinapyl alcohol derivative formation. Phytochemistry, 66: 2072-2091.

Danielsson, M., Lundén, K., Elfstrand, M., Hu, J., Zhao, T., Arnerup, J., Ihrmark, K., Swedjemark, G., Borg-Karlson, A.K., \& Stenlid, J. (2011). Chemical and transcriptional responses of Norway spruce genotypes with different susceptibility to Heterobasidion spp. infection. BMC Plant Biology, 11, 154.

D'Auria, J.C. (2006). Acyltransferases in plants: a good time to be BAHD. Current Opinion in Plant Biology, 9, 331-340.

De Clerck, O., Kao, S.-M., Bogaert, K. A., Blomme, J., Foflonker, F., Kwantes, M. ... Bothwell, J. H. (2018) Insights into the evolution of multicellularity from the sea lettuce genome. Current Biology, 28, 2921-2933.e2925. 
1159 Delwiche, C. F., Graham, L. E., Thomson, N. (1989). Lignin-like compounds and $1160 \quad$ sporopollenin in Coleochaete, an algal model for land plant ancestry. Science, 245, 3991161401.

1162 Devic, M., Guilleminot, J., Debeaujon, I., Bechtold, N., Bensaude, E., Koornneef, M., 1163 Pelletier, G. \& Delseny, M. (1999). The BANYULS gene encodes a DFR $\square$ like protein 1164 and is a marker of early seed coat development. The Plant Journal, 19, 387-398.

1165 de Azevedo Souza, C., Kim, S.S., Koch, S., Kienow, L., Schneider, K., McKim, S.M., 1166 Haughn, G.W., Kombrink, E., \& Douglas, C.J. (2009). A novel fatty acyl-CoA synthetase is required for pollen development and sporopollenin biosynthesis in Arabidopsis. Plant Cell 21, 507-525.

de Vries, J., Curtis, B.A., Gould, S.B. \& Archibald, J.M. (2018). Embryophyte stress signaling evolved in the algal progenitors of land plants. Proceedings of the National Academy of Sciences USA, 115, E3471-E3480.

de Vries, J., de Vries, S., Slamovits, C.H., Rose, L.E. \& Archibald, J.M. (2017) How embryophytic is the biosynthesis of phenylpropanoids and their derivatives in streptophyte algae? Plant Cell Physiol 58, 934-945.

de Vries, J., \& Archibald, J.M. (2018) Plant evolution: Landmarks on the path to terrestrial life. New Phytologist 217, 1428-1434.

de Vries, J. de Vries, S., Curtis, B. A., Zhou, H., Penny, S., Feussner, K., Pinto, D. M., Steinert, M., Cohen, A. M., von Schwartzenberg, K., \& Archibald, J. M. (2020) Heat stress response in the closest algal relatives of land plants reveals conserved stress signalling circuits. The Plant Journal, 103, 1025-1048.

de Vries, J., \& Ischebeck, T. (2020) Ties between stress and lipid droplets pre-date seeds. Trends Plant Sci. 12, 1203-1214.

de Vries, S., Herrfurth, C., Li, F.-W., Feussner, I., \& de Vries, J. (2021) An ancient route towards salicylic acid and its implications for the perpetual Trichormus-Azolla

Dixon, R. A., \& Paiva, N. L. (1995) Stress-induced phenylpropanoid metabolism. Plant Cell,

1188 Dixon, R.A., Achnine, L., Kota, P., Liu, C.-J., Srinivasa Reddy, M.S., \& Wang, L. (2002).

1189 The phenylpropanoid pathway and plant defence-a genomics perspective. Molecular $1190 \quad$ Plant Pathology 3, 371-390.

1191 Do, C.-T., Pollet, B., Thévenin, J., Sibout, R., Denoue, D., Barrière, Y., Lapierre, C., \& 1192 Jouanin, L. (2007) Both caffeoyl coenzyme A 3-O-methyltransferase 1 and caffeic 
1193

1194

1195

1196

1197

1198

1199

1200

1201

1202

1203

1204

1205

1206

1207

1208

1209

1210

1211

1212

1213

1214

1215

1216

1217

1218

1219

1220

1221

1222

1223

1224

acid $O$-methyltransferase 1 are involved in redundant functions for lignin, flavonoids and sinapoyl malate biosynthesis in Arabidopsis. Planta, 226, 1117-1129.

Ehlting, J., Büttner, D., Wang, Q., Douglas, C.J., Somssich, I.E., \& Kombrink, E. (1999). Three 4-coumarate:coenzyme A ligases in Arabidopsis thaliana represent two evolutionarily divergent classes in angiosperms. The Plant Journal, 19, 9-20.

Emiliani, G., Fondi, M., Fani, R. \& Gribaldo, S. (2009). A horizontal gene transfer at the origin of phenylpropanoid metabolism: a key adaptation of plants to land. Biology Direct, 4, 7.

Eudes, A., Pereira, J.H., Yogiswara, S., Wang, G., Benites, V.T., Baidoo, E.E.K., Lee, T.S., Adams, P.D., Keasling, J.D., \& Loqué, D. (2016) Exploiting the substrate promiscuity of hydroxycinnamoyl-CoA: shikimate hydroxycinnamoyl transferase to reduce lignin. Plant Cell Physiol, 57, 568-579.

Fellenberg, C., Milkowski, C., Hause, B., Lange, P. $\square$ R., Böttcher, C., Schmidt, J., \& Vogt, T. (2008). Tapetum $\square$ specific location of a cation $\square$ dependent $\mathrm{O} \square$ methyltransferase in Arabidopsis thaliana. The Plant Journal, 56, 132- 145.

Franks, N.P., Jenkins, A., Conti, E., Lieb, W.R., \& Brick, P. (1998). Structural basis for the inhibition of firefly luciferase by a general anesthetic. Biophysical Journal, 75, 22052211.

Ferrer, J.L., Zubieta, C., Dixon, R.A., \& Noel, J.P. (2005). Crystal structures of alfalfa caffeoyl coenzyme A 3-O-methyltransferase. Plant Physiology, 137, 1009-1017.

Fürst-Jansen, J.M.R., de Vries, S. \& de Vries, J. (2020). Evo-physio: on stress responses and the earliest land plants. Journal of Experimental Botany, 11, 3254-3269.

Goiris, K., Muylaert, K., Voorspoels, S., Noten, B., De Paepe, D., Baart, G. J. E., \& De Cooman, L. (2014). Detection of flavonoids in microalgae from different evolutionary lineages. Journal of Phycology, 50, 483-492.

Gouy, M., Guindon, S., \& Gascuel, O. (2010) SeaView Version 4: A multiplatform graphical user interface for sequence alignment and phylogenetic tree building. Molecular Biology and Evolution, 27, 221-224.

Güngör, E., Brouwer P., Dijkhuizen, L. W., Shaffar, D. C., Nierop, K. G. J., de Vos, R. C. H., Toraño, J. S., van der Meer, I. N., \& Schluepmann, H. (2021) Azolla ferns testify: seed plants and ferns share a common ancestor for leucoanthocyanidin reductase enzymes. New Phytologist, 229, 1118-1132. 
1225

1226

Guo, D.-M., Ran, J.-H., \& Wang, X.-Q. (2010). Evolution of the Cinnamyl/Sinapyl Alcohol Dehydrogenase (CAD/SAD) Gene Family: The Emergence of Real Lignin is Associated with the Origin of Bona Fide CAD. Journal of Molecular Evolution, 71, 202-218.

Grienenberger, E., Kim, S. S., Lallemand, B., Geoffroy, P., Heintz, D., de Azevedo Souza, C., Heitz, T., Douglas, C. J., Legrand, M. (2010) Analysis of TETRAKETIDE $\alpha-$ PYRONE REDUCTASE function in Arabidopsis thaliana reveals a previously unknown, but conserved, biochemical pathway in sporopollenin monomer biosynthesis. The Plant Cell 22, 4067-4083.

Gross, G. G., Stöckigt, J., Mansell, R. L., \& Zenk, M. H. (1973). Three novel enzymes involved in the reduction of ferulic acid to coniferyl alcohol in higher plants: ferulate: CoA ligase, feruloyl-CoA reductase and coniferyl alcohol oxidoreductase. FEBS Lett. 31, 283-286

Ha, C.M., Escamilla-Trevino, L., Yarce, J.C.S., Kim, H., Ralph, J., Chen, F., \& Dixon, R.A. (2016). An essential role of caffeoyl shikimate esterase in monolignol biosynthesis in Medicago truncatula. The Plant Journal, 86, 363-375.

Hamberger, B., Ellis, M., Friedmann, M., de Azevedo Souza, C., Barbazuk, B., \& Douglas, C.J. (2007). Genome-wide analyses of phenylpropanoid-related genes in Populus trichocarpa, Arabidopsis thaliana, and Oryza sativa: the Populus lignin toolbox and conservation and diversification of angiosperm gene families. Canadian Journal of Botany, 85, 1182-1201.

Hoffmann, L., Maury, S., Martz, F., Geoffroy, P., \& Legrand, M. (2003). Purification, cloning, and properties of an acyltransferase controlling shikimate and quinate ester intermediates in phenylpropanoid metabolism. The Journal of Biological Chemistry, 278, 95-103.

Hori, K., Maruyama, F., Fujisawa, T., Togashi, T., Yamamoto, N., Seo, M., ... Ohta, H. (2014). Klebsormidium flaccidum genome reveals primary factors for plant terrestrial adaptation. Nature Communications, 5, 3978.

Hu, Y., Gai, Y., Yin, L., Wang, X., Feng, C., Feng, L., Li, D., Jiang, X.N., \& Wang, D.C. (2010). Crystal structures of a Populus tomentosa 4-coumarate:CoA ligase shed light on its enzymatic mechanisms. The Plant Cell, 22, 3093-3104.

Huerta-Cepas, J., Serra, F., \& Bork, P. ETE 3: Reconstruction, analysis, and visualization of phylogenomic data. Molecular Biology and Evolution, 33, 1635-1638.

Jahns, P., \& Holzwarth, A.R. (2012). The role of the xanthophyll cycle and of lutein in photoprotection of photosystem II. BBA - Bioenergetics, 1817, 182-193. 
1259 Jiao, C., Sørensen, I., Sun, X., Sun, H., Behar, H., Alseekh, S., ... Rose, J. K. C. (2020). The Penium margaritaceum genome: hallmarks of the origins of land plants. Cell, 181, P1097-1111.E12

1262 Jones, P., Binns, D., Chang, H.-Y., Fraser, M., Li, W., McAnulla, C., ... Hunter, S. (2014). 1263 InterProScan 5: genome-scale protein function classification. Bioinformatics, 30, 1236$1264 \quad 1240$.

1265 Ju, C., Van de Poel, B., Cooper, E. D., Thierer, J. H., Gibbons, T. R., Delwiche, C. F., \& Chang, C. (2015). Conservation of ethylene as a plant hormone over 450 million years of evolution. Nature Plants, 1, 14004.

Kaur, H., Heinzel, N., Schöttner, M., Baldwin, I.T., \& Gális, I. (2010). R2R3-NaMYB8 regulates the accumulation of phenylpropanoid-polyamine conjugates, which are essential for local and systemic defense against insect herbivores in Nicotiana attenuata. Plant Physiology, 152, 1731-1747.

Kim, S.-J., Kim, M.-R., Bedgar, D.L., Moinuddin, S.G.A., Cardenas, C.L., Davin, L.B., Kang, C., \& Lewis, N.G. (2004). Functional reclassification of the putative cinnamyl alcohol dehydrogenase multigene family in Arabidopsis. Proceedings of the National Academy of Sciences USA, 101, 1455-1460.

Kim, R. J., Kim, H.J., Shim, D., \& Suh, M.C. (2016). Molecular and biochemical characterizations of the monoacylglycerol lipase gene family of Arabidopsis thaliana. The Plant Journal, 85, 758-771.

König, S., Feussner, K., Kaever, A., Landesfeind, M., Thurow, C., Karlovsky, P., Gatz, C., response of Arabidopsis against Verticillium longisporum. New Phytologist, 202, 823837.

Kriegshauser, L., Knosp, S., Grienenberger, E., Tatsumi, K., Gütle, D. D., Sørensen, I., ... Renault, H. (2021) Function of the HYDROXYCINNAMOYL-CoA:SHIKIMATE HYDROXYCINNAMOYL TRANSFERASE is evolutionarily conserved in embryophytes. The Plant Cell, in press, preprint on bioRxiv, 2020.09.16.300285.

Labar, G., Bauvois, C., Borel, F., Ferrer, J. $\square$ L., Wouters, J., \& Lambert, D.M. (2010), Crystal Structure of the Human Monoacylglycerol Lipase, a Key Actor in Endocannabinoid Signaling. ChemBioChem, 11, 218-227.

Labeeuw, L., Martone, P.T., Boucher, Y., \& Case, R.J. (2015) Ancient origin of the biosynthesis of lignin precursors. Biology Direct 10, 23. 
1292 Lacombe, E., Hawkins, S., Doorsselaere, J.V., Piquemal, J., Goffner, D., Poeydomenge, O., 1293 Boudet, A-M., \& Grima-Pettenati, J. G. (1997). Cinnamoyl CoA reductase, the first committed enzyme of the lignin branch biosynthetic pathway: cloning, expression and phylogenetic relationships. The Plant Journal 11, 429-441.

1297

1298

Lang, D., Ullrich, K. K., Murat, F., Fuchs, J., Jenkins, J., Haas, F. B., ... Rensing, S. A. (2018) The Physcomitrella patens chromosome-scale assembly reveals moss genome structure and evolution. The Plant Journal, 93, 515-533.

Lamesch, P., Berardini, T. Z., Li, D., Swarbreck, D., Wilks, C., Sasidharan, R., ... Huala, E. (2011). The Arabidopsis Information Resource (TAIR): improved gene annotation and new tools. Nucleic Acids Research, 40, D1202-D1210.

Le S.Q, \& Gascuel O. (2008). An Improved General Amino Acid Replacement Matrix. Mol. Biol. Evol. 25, 1307-1320.

Leebens-Mack, J.H., Barker, M.S., Carpenter, E.J., Deyholos, M. K., Gitzendanner, M. A., Graham, S. W., ... Wong, G. K.-S. (2019) One thousand plant transcriptomes and the phylogenomics of green plants. Nature, 574, 679-685.

Letunic, I., \& Bork, P. (2019). Interactive Tree Of Life (iTOL) v4: recent updates and new

Li, W., Zhang, F., Wu, R., Jia, R., Li, G., Guo, Y., Liu, C., \& Wang, W. (2017). A novel NMethyltransferase in Arabidopsis appears to feed a conserved pathway for nicotinate detoxification among land plants and is associated with lignin biosynthesis. Plant Physiology, 174, 1492-1504.

Li, F.-W., Nishiyama, T., Waller, M., Frangedakis, E., Keller, J., Li, Z. ... Szövévyi, P. (2020) Anthoceros genomes illuminate the origin of land plants and the unique biology of hornworts. Nature Plants, 6, 259-272.

Li, F.-W., Brouwer, P., Carretero-Paulet, L., Cheng, S., de Vries, J., Delaux, P.-M., ... Pryer, K. M. (2018) Fern genomes elucidate land plant evolution and cyanobacterial symbioses. Nature Plants, 4, 460-472.

Louie, G. V., Bowman, M. E., Tu, Y., Mouradov, A., Spangenberg, G., \& Noel, J.P. (2010). Structure $\square$ function analyses of a caffeic acid $O \square$ methyltransferase from perennial ryegrass reveal the molecular basis for substrate preference. The Plant Cell, 22, 4114- 4127.

Maeda, H. A., \& Fernie, A. R. (2021) Evolutionary history of plant metabolism. Annual Review of Plant Biology, 72, 10.1146/annurev-arplant-080620-031054 
1325 Mansell, R. L. G., Gross, G. G., Stöckigt, J., Franke, H., \& Zenk, M. H. (1974) Purification and properties of cinnamyl alcohol dehydrogenase from higher plants involved in lignin biosynthesis. Phytochemistry, 13, 2427-2435.

Matsuno, M., Compagnon, V., Schoch, G.A., Schmitt, M., Debayl, D., Bassard, J.-E. ... Werck-Reichhart, D. (2009). Evolution of a novel phenolic pathway for pollen development. Science 325, 1688-1692.

Martone, P. T., Estevez, J. M., Lu, F., Ruel, K., Denny, M. W., Somerville, C., \& Ralph, J. (2009). Discovery of lignin in seaweed reveals convergent evolution of cell-wall architecture. Current Biology, 19, 169-175.

Martz, F., Maury, S., Pincon, G., \& Legrand, M. (1998). cDNA cloning, substrate specificity and expression study of tobacco caffeoyl- CoA 3-O-methyltransferase, a lignin biosynthetic enzyme. Plant Molecular Biology, 36, 427-437.

Maury, S., Geoffroy, P., \& Legrand, M. (1999). Tobacco O-methyltransferases involved in phenylpropanoid metabolism: the different caffeoyl- coenzyme A/5-hydroxyferuloylcoenzyme A 3/5-O-methyltransferase and caffeic acid/5-hydroxyferulic acid 3/5-Omethyltransferase classes have distinct substrate specificities and expression patterns. Plant Physiology, 121, 215-224.

Merchant, S. S., Prochnik, S. E., Vallon, O., Harris, E. H., Karpowicz, S. J., Witman, G. B., ... Grossman, A. R. (2007). The Chlamydomonas genome reveals the evolution of key animal and plant functions. Science 318, 245-250.

Miller, M., Owens, S.J., \& Rørslett, B. (2011). Plants and colour: flowers and pollination. Optics \& Laser Technology, 43, 282-294.

Moffitt, M.C., Louie, G.V., Bowman, M.E., Pence, J., Noel, J.P., \& Moore, B.S. (2007). Discovery of two cyanobacterial phenylalanine ammonia lyases: kinetic and structural characterization. Biochemistry, 46, 1004-1012.

Moreau, H., Verhelst, B., Couloux, A., Derelle, E., Rombauts, S., Grimsley, N., ... Vandepoele, K. (2012). Gene functionalities and genome structure in Bathycoccus prasinos reflect cellular specializations at the base of the green lineage. Genome Biology, $13, \mathrm{R} 74$. (2006). Structural basis for the spectral difference in luciferase bioluminescence. Nature, $440,372-376$.

Nelson, D., \& Werck-Reichhart, D. (2011). A P450-centric view of plant evolution. The Plant Journal, 66, 194-211. 
1359 Nishiyama, T., Sakayama, H., de Vries, J., Buschmann, H., Saint-Marcoux, D., Ullrich, K. 1360 K., ... Rensing, S. A. (2018). The Chara genome: secondary complexity and 1361 implications for plant terrestrialization. Cell, 174, 448-464.

1362 Nystedt, B., Street, N. R., Wetterbom, A., Zuccolo, A., Lin, Y.-C., Scofield, D. G., ... 1363 Jansson, S. (2013). The Norway spruce genome sequence and conifer genome evolution. $1364 \quad$ Nature, 497, 579-584.

1365 Oliva, J., Rommel, S., Fossdal, C.G., Hietala, A.M., Nemesio-Gorriz, M., Solheim, H., \& 1366 Elfstrand, M. (2015). Transcriptional responses of Norway spruce (Picea abies) inner sapwood against Heterobasidion parviporum. Tree Physiology, 35, 1007-1015.

Omura, T. (1999). Forty years of cytochrome P450. Biochemical and Biophysical Research Communications, 266, 690-698.

Ouyang, S., Zhu, W., Hamilton, J., Lin, H., Campbell, M., Childs, K., ... Buell, C. R. (2007). The TIGR Rice Genome Annotation Resource: improvements and new features. Nucleic Acids Research, 35, D883-D887.

Overdijk, E.J.R., de Keijzer, J., de Groot, D., Schoina, C., Bouwmeester, K., Ketelaar, T., \& Govers, F. (2016). Interaction between the moss Physcomitrella patens and Phytophthora: a novel pathosystem for live $\square$ cell imaging of subcellular defence. Journal of Microscopy, 263, 171-180.

Palenik, B., Grimwood, J., Aerts, A., Rouzé, P., Salamov, A., Putman, N., ... Grigoriev, I. V. (2007). The tiny eukaryote Ostreococcus provides genomic insights into the paradox of plankton speciation. Proceedings of the National Academy USA. 104, 7705-7710.

Pan, H., Zhou, R., Louie, G.V., Mühlemann, J. K., Bomati, E. K., Bowman, M. E., ... Wang, X. (2014). Structural Studies of Cinnamoyl-CoA Reductase and Cinnamyl-Alcohol Dehydrogenase, key enzymes of monolignol biosynthesis. The Plant Cell 26, 3709.

Piatkowski, B. T., Imwattana, K., Tripp, E. A., Weston, D. J., Healey, A., Schmutz, J., \& Shaw, A. J. (2020). Phylogenomics reveals convergent evolution of red-violet coloration in land plants and the origins of the anthocyanin biosynthetic pathway. Molecular Phylogenetics and Evolution, 151, 106904.

Ponce De León, I., Schmelz, E. A., Gaggero, C., Castro, A., Álvarez, A., \& Montesano, M. (2012). Physcomitrella patens activates reinforcement of the cell wall, programmed cell death and accumulation of evolutionary conserved defence signals, such as salicylic acid and 12-oxo-phytodienoic acid, but not jasmonic acid, upon Botrytis cinerea infection. Molecular Plant Pathology, 13, 960-74. 
1392 Prochnik, S.E., Umen, J., Nedelcu, A. M., Hallmann, A., Miller, S. M., Nishii, I., ... Rokhsar,

1393 D. S. (2010). Genomic analysis of organismal complexity in the multicellular green alga $1394 \quad$ Volvox carteri. Science, 329, 223-226

1395 Puttick, M.N., Morris, J.L., Williams, T.A., Cox, C.J., Edwards, D., Kenrick, P., Pressel, S., 1396 Wellman, C.H., Schneider, H., Pisani, D., \& Donoghue, P.C.J. (2018). The 1397 interrelationships of land plants and the nature of the ancestral embryophyte. Current 1398 Biology, 28, 733-745.

1399 Ralph, J., Lundquist, K., Brunow, G., Lu, F., Kim, H., Schatz, P. F., Marita, J. M., Hatfield, 1400 R. D., Ralph, S. A., Christensen, J. H., \& Boerjan, W. (2004). Lignins: natural polymers from oxidative coupling of 4-hydroxyphenylpropanoids. Phytochemistry Reviews, 3, 2960.

Rupasinghe S., Baudry J. \& Schuler M.A. (2003) Common active site architecture and binding strategy of four phenylpropanoid P450s from Arabidopsis thaliana as revealed by molecular modeling. Protein Engineering Design and Selection, 16, 721-731.

Renault, H., De Marothy, M., Jonasson, G., Lara, P., Nelson, D. R., Nilsson, I., André, F., von Heijne, G., \& Werck-Reichhart, D. (2017b). Gene duplication leads to altered membrane topology of a cytochrome P450 enzyme in seed plants. Molecular Biology and Evolution, 34, 2041-2056.

1413 Renault, H., Werck-Reichhart, D., \& Weng, J-K. (2019). Harnessing lignin evolution for biotechnological applications. Current Opinion in Biotechnology, 56, 105-111.

Rensing, S.A. (2014). Gene duplication as a driver of plant morphogenetic evolution. Current Opinion in Plant Biology, 17, 43-48.

Rensing, S.A. (2018) Great moments in evolution: the conquest of land by plants. Current Opinion in Plant Biology, 42, 49-54.

Rippin, M., Becker, B. and Holzinger, A. (2017) Enhanced desiccation tolerance in mature cultures of the streptophytic green alga Zygnema circumcarinatum revealed by transcriptomics. Plant \& Cell Physiology, 58, 2067-2084. Metatranscriptomic and metabolite profiling reveals vertical heterogeneity within a Zygnema green algal mat from Svalbard (High Arctic). Environmental Microbiology, 21, 4283-4299. 
1426 Ro, D. K., Mah, N., Ellis, B. E., \& Douglas, C. J. (2001). Functional characterization and subcellular localization of poplar (Populus trichocarpa x Populus deltoides) cinnamate 4-hydroxylase. Plant Physiology, 126, 317-329.

1429 Russell, D.W., Conn, E.E. (1967). The cinnamic acid 4-hydroxylase from pea seedlings. $1430 \quad$ Archives of Biochemistry and Biophysics 122, 256-258.

1431 Saballos, A., Ejeta, G., Sanchez, E., Kang, C., \& Vermerris, W. (2009) A genomewide 1432 analysis of the cinnamyl alcohol dehydrogenase family in sorghum [Sorghum bicolor (L.) Moench] identifies SbCAD2 as the brown midrib6 gene. Genetics, 181, 783-795.

Saito, N., \& Harborne, J.B. (1992). Correlations between anthocyanin type, pollinator and flower colour in the labiatae. Phytochemistry, 31, 3009-3015.

Scheres, B., \& van der Putten, W.H. (2017) The plant perceptron connects environment to development. Nature 543, 337-345.

Schoenbohm, C., Martens, S., Eder, C., Forkmann, G., \& Weisshaar, B. (2000) Identification of the Arabidopsis thaliana flavonoid 3'-hydroxylase gene and functional expression of the encoded P450 enzyme. Biological Chemistry, 381, 749-753.

Sheahan, J.J. (1996) Sinapate esters provide greater UV-B attenuation than flavonoids in Arabidopsis thaliana (Brassicaceae). American Journal of Botany, 83, 679-686.

Sheehan, H., Hermann, K., \& Kuhlemeier, C. (2012). Color and Scent: How Single Genes

1446 Shockey, J.M., Fulda, M.S., \& Browse, J. (2003). Arabidopsis contains a large superfamily of 1447 Acyl-Activating Enzymes. Phylogenetic and biochemical analysis reveals a new class of 1448 Acyl-Coenzyme A Synthetases. Plant Physiology, 132, 1065-1076.

1449 Sierro, N. Battey, J., Ouadi, S., Bakaher, N., Bovet, L., Willig, A., Goepfert, S., Peitsch, M. Influence Pollinator Attraction. Cold Spring Harbor Symposia on Quantitative Biology, C., \& Ivanov, N. V. (2014). The tobacco genome sequence and its comparison with those of tomato and potato. Nature Communications, 5, 3833.

Slotte, T., Hazzouri, K. M., Ågren, J. A., Koenig, D., Maumus, F., Guo, Y.-L., ... Wrigth, S. I. (2013). The Capsella rubella genome and the genomic consequences of rapid mating system evolution. Nature Genetics, 45, 831-835

Sørensen, I., Pettolino, F.A., Bacic, A., Ralph, J., Lu, F., O’Neill, M.A., Fei, Z., Rose, J.K.C., Domozych, D.S., Willats, W.G.T. (2011). The charophycean green algae provide insights into the early origins of plant cell walls. The Plant Journal, 68, 201-211.

Suzuki, S., \& Umezawa, T. (2007). Biosynthesis of lignans and norlignans. Journal of Wood Science 53, 273-284. 
1460 Sytar, O., Zivcak, M., Bruckova, K., Brestic, M., Hemmerich, I., Rauh, C., Simko, I. 2018.

1461 Shift in accumulation of flavonoids and phenolic acids in lettuce attributable to changes

1462 in ultraviolet radiation and temperature. Scientia Horticulturae, 239, 193-204.

1463 Szövényi, P., Frangedakis, E., Ricca, M., Quandt, D., Wicke, S., Langdale, J.A. (2015).

1464 Establishment of Anthoceros agrestis as a model species for studying the biology of 1465 hornworts. BMC Plant Biology, 15, 1-7.

1466 Szövényi, P., Gunadi, A., Li, F.-W. (2021). Charting the genomic landscape of seed-free 1467 plants. Nature Plants, https://doi.org/10.1038/s41477-021-00888-z

1468 Tan, D.-X., Hardeland, R., Manchester, L.C., Korkmaz, A., Ma, S., Rosales-Corral, S., 1469 Reiter, R.J. (2012). Functional roles of melatonin in plants, and perspectives in nutritional and agricultural science. Journal of Experimental Botany, 63, 577-597.

Tanaka, A., Shigemitsu, T., Yokota, Y., Shika, N. (1997). A new Arabidopsis mutant induced synthesis with spotted pigmentation. Genes \& Genetic Systems, 72, 141-148.

The International Brachypodium Initiative. (2010). Genome sequencing and analysis of the model grass Brachypodium distachyon. Nature, 463, 763-768.

Urban, P., Werck-Reichhart, D., Teutsch, H.G., Durst, F., Regnier, S., Kazmeier, M., \& Pompon, D. (1994). Characterization of recombinant plant cinnamate 4-hydroxylase produced in yeast. Kinetic and spectral properties of the major plant P450 of the phenylpropanoid pathway. European Journal of Biochemistry, 222, 843-850.

Vanholme, R., Storme, V., Vanholme, B., Sundin, S., Christensen, J.H., Goemine, G., Halpin, C., Rohde, A., Morreel, K., Boerjan, W. (2012) A systems biology view of responses to lignin biosynthesis perturbations in Arabidopsis. The Plant Cell, 24, 35063529.

Vanholme, R., Cesarino, I., Rataj, K., Xiao, Y., Sundin, L., Goeminne, G., ... Boerjan, W. (2013). Caffeoyl shikimate esterase (CSE) is an enzyme in the ligninbiosynthetic pathway in Arabidopsis. Science, 341, 1103-1106.

Vanholme, R., De Meester, B., Ralph, J., \& Boerjan, W. (2019). Lignin biosynthesis and its integration into metabolism. Current Opinion Biotechnology, 56, 230-239.

Vogt, T. (2010). Phenylpropanoid biosynthesis. Molecular Plant, 3, 2-20.

Wan, T. et al. (2018). A genome for gnetophytes and early evolution of seed plants. Nature Plants, 4, 82-89.

Wang, S., Li, L., Li, H., Sahu, S. K., Wang, H., Xu, Y., ... Liu, X. (2020) Genomes of earlydiverging streptophyte algae shed light on plant terrestrialization. Nature Plants, 6, 95106. 
1494 Weng, J.-K., Li, X., Stout, J., \& Chapple, C. (2008) Independent origins of syringyl lignin in vascular plants. Proceedings of the National Academy of Sciences USA, 105, 7887-7892.

Weng, J.-K., \& Chapple, C. (2010) The origin and evolution of lignin biosynthesis. New Phytologist, 187, 273-285.

1498 Weng, J.-K., Akiyama, T., Ralph, J., \& Chapple, C. (2011) Independent recruitment of an O1499 methyltransferase for syringyl lignin biosynthesis in Selaginella moellendorffii. The $1500 \quad$ Plant Cell, 23, 2708-2724.

Weng, J. K. (2013) The evolutionary paths towards complexity: a metabolic perspective. New Phytologist, 201, 1141-1149.

Whelan, S., \& Goldman, N. (2001). A general empirical model of protein evolution derived from multiple protein families using a maximum-likelihood approach. Molecular Biology and Evolution, 18, 691-699.

Wickett, N.J., Mirarab, S., Nguyen, N., Warnow, T., Carpenter, E., Matasci, N., ... LeebensMack, J. (2014) Phylotranscriptomic analysis of the origin and early diversification of land plants. Proceedings of the National Academy of Sciences USA, 111, E4859-E4868.

Wohl, J., \& Petersen, M. (2020). Functional expression and characterization of cinnamic acid $4 \square$ hydroxylase from the hornwort Anthoceros agrestis in Physcomitrella patens. Plant Cell Reports, 39, 597-607.

Wolf, L., Rizzini, L., Stracke, R., Ulm, R., \& Rensing, S. A. (2010) The molecular and physiological repsonses of Physcomitrella patens to ultraviolet-B radiation. Plant Physiol. 153, 1123-1134.

Xu, Z., Zhang, D., Hu, J., Zhou, X., Ye, X., Reichel, K. L., ... Yuan, J. S. (2009). Comparative genome analysis of lignin biosynthesis gene families across the plant kingdom. BMC Bioinformatics 10, S3. plants. Molecular Plant 13, 1644-1653. 
1527 Yang, J., Yan, R., Roy, A., Xu, D., Poisson, J., \& Zhang, Y. (2015). The I-TASSER Suite: protein structure and function prediction. Nature Methods 12, 7-8.

Ye, Z.-H., Kneusel, R.E., Matern, U., \& Varner, J. E. (1994). An alternative methylation pathway in lignin biosynthesis in Zinnia. The Plant Cell, 6, 1427-1439.

Ye, Z.-H., \& Varner, J.E. (1995). Differential expression of two $O$-methyltransferases in 1532 lignin biosynthesis in Zinnia elegans. Plant Physiology, 108, 459-467.

Youn, B., Camacho, R., Moinuddin, S.G.A., Lee, C., Davin, L.B., Lewis, N.G., ..., (2006) Crystal structures and catalytic mechanism of the Arabidopsis cinnamyl alcohol dehydrogenases AtCAD5 and AtCAD4. Organic and Biomolecular Chemistry, 4, 16871697.

Zhang, Y. (2008). I-TASSER server for protein 3D structure prediction. BMC Bioinformatics, 9,40 .

Zhang, J., Fu, X.-X., Li, R.-Q., Zhao, X., Liu, Y., Li, M.-H., ... Chen, Z.-D. (2020). The hornwort genome and early land plant evolution. Nature Plants, 6, 107-118. modulation of lignin monomer methylation by caffeic acid/5-hydroxyferulic acid 3/5-Omethyltransferase. The Plant Cell, 14, 1265-1277. 
Phe $\stackrel{\mathrm{PAL}}{\longrightarrow}$ Cinnamate $\stackrel{\mathrm{C} 4 \mathrm{H}}{\longrightarrow} \rightarrow p$-Coumarate

ACoS, 尚

ACos, 自 stilbenes

$\mathrm{C} 4 \mathrm{H}$

4CL

Caffeate - - $\square-D$ Ferulate -

CCR
!'

Cinnamaldehyde
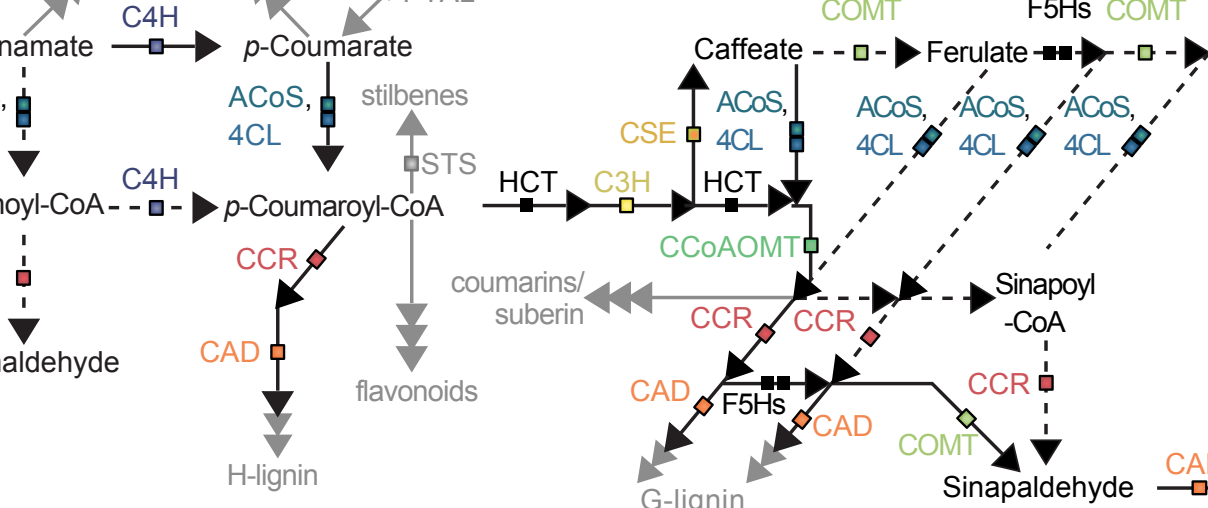

S-lignin 
96

82

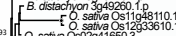

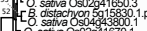

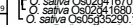

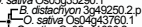

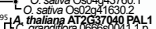

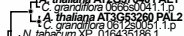

in.

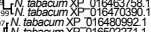

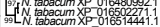

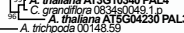

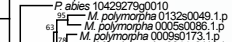

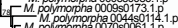

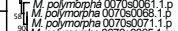

- M. pohymorpha 001450211.1.

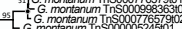

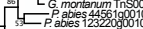

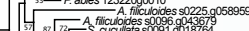

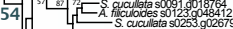

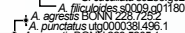

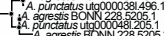

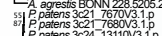

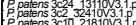

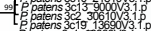

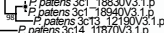

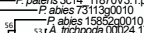

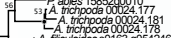

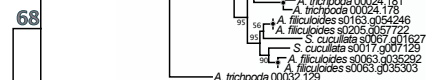

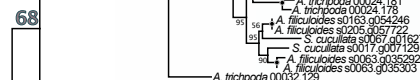

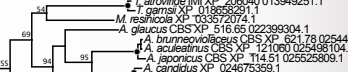

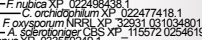

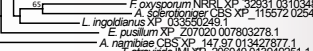

85

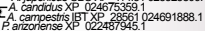

${ }^{61}{ }^{61}$

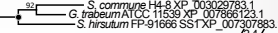

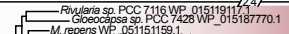

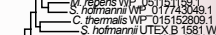

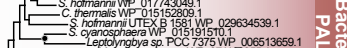

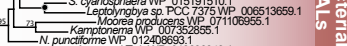

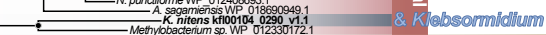
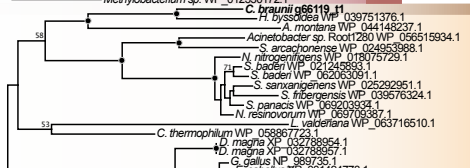

- i. magna XP 032789957.1

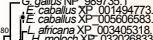

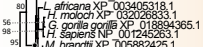

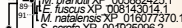

R. bivitatum XP 020255906.21

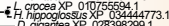

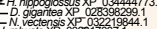

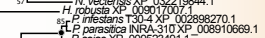

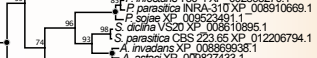

A. astaci XP 00982743331.

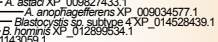

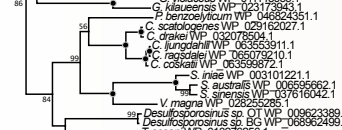

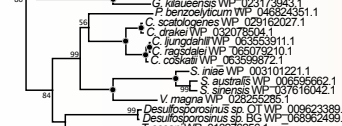

- . sculloturatus XP 0232289101

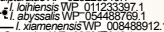

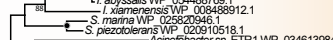

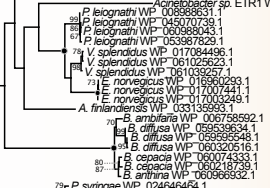

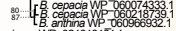

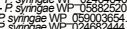

P s singae W

M buynazense WP 024699180.

C. M. kouniigamae 935530 t1

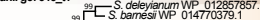

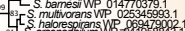

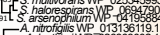

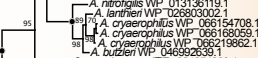

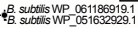


Substrate binding

$\downarrow$ Enzymatic function

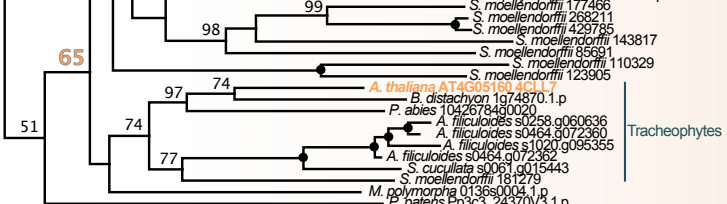

98 moellendortfliil111112

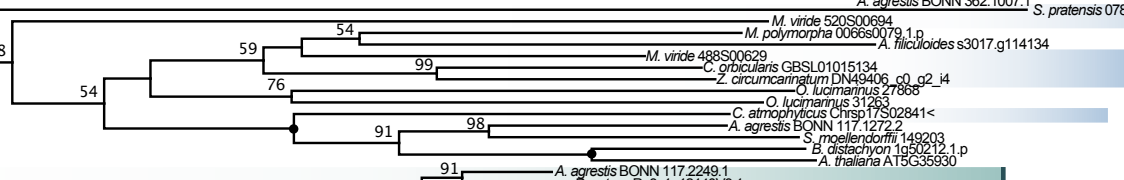

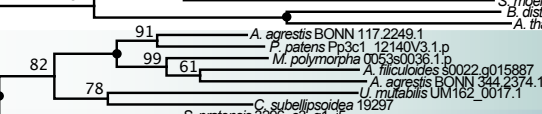

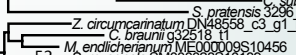
$\left[\begin{array}{ccc}4 \\ 52 \\ 52\end{array}\right.$

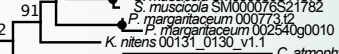

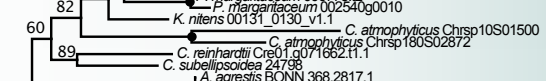

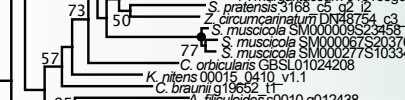

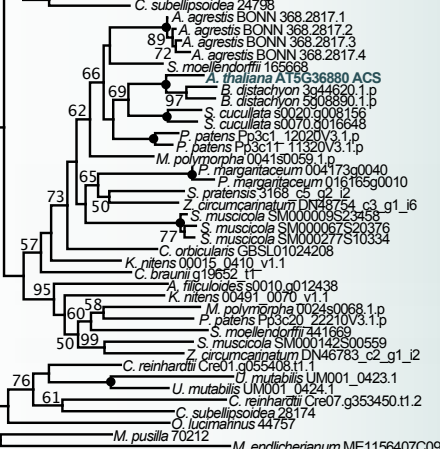


$\checkmark$ Substrate binding pocket

* NADP+ binding

- $\mathrm{Zn}^{2+}$ binding 
- G. Montanum Insontanum Ins000145195103

- A. thaliana AT1G74540 CYP98A8 A. thaliana AT1G7450250 CY.1.p

tabacum XP 01644317:

tabacum $\times 1-01643318: 1$

tabacum X $\times 1646949.1$

N. tabacum XP 016479719.1

A. thaliana AT2G40890 C3H (CYP98A3)

99 2 . C. grandiffora 1642s0055.1.

iN. tabacum XP 016482834

- B. distachyon 2921300.1 .

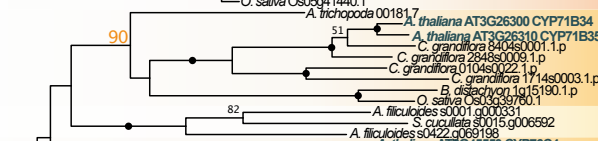

A. thalliana AT2 G45550 CYP76C4

99 A. thaliana AT2 G45560 CYP76C1 C. grandifiora 0698s0005.1.p - G. mandifiora 0698 so004.1.12

S. cucullata s0074.9017295

A. thaliana AT5G07990 CYP75B1/TT7

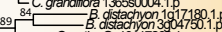

O. sativa Os10g17260.1

$\rightarrow-B$ distachyon 401050.1 .

- B. distachyon 1957000.1 . B p

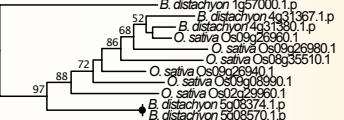

Monocots

91 A. trichopoda 00066.179

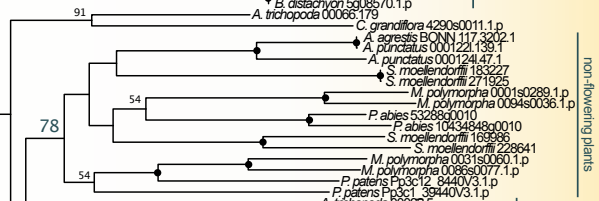

87

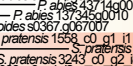
agrestis BONN 369.483

A. puinctatus 000148.58 .1

$94 \quad$ E. Distactyon $3914390.1 . \mathrm{p}$

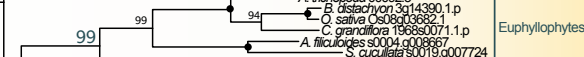

S. cucullatas s019.9007724

\begin{tabular}{l|l} 
& \\
pha 019750009.1.p & Bryophytes
\end{tabular} If Anthoceros sp. CYP 450 s
72 .

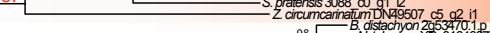

98 N tabacum XP 016468779.1

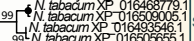

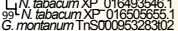

- K nitentens 0000350080 v1.1

Spermatophytes

CYP450s

\section{Embryophytes} $K$ niter

C. braunï $92943 \mathrm{t} 1$

C. brauning12412 $\mathrm{t1}$

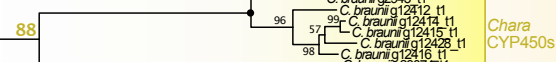

C. braunig 12416 t1 

V Substrate binding pocket

$\downarrow$ Enzymatic function

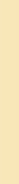

SS. moollendorfitis 85242

Selaginella COMT

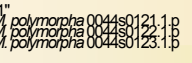

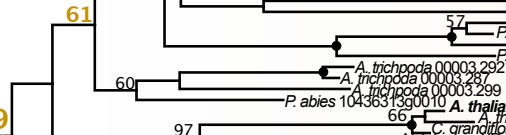

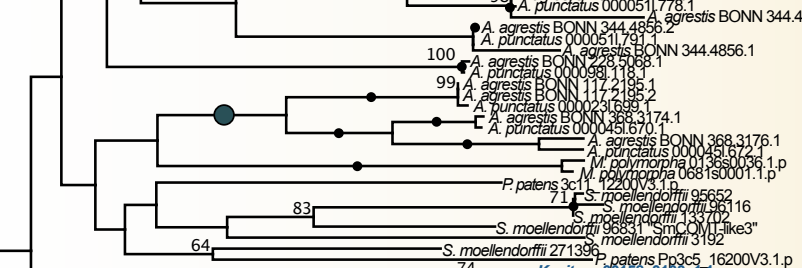

Hornworts

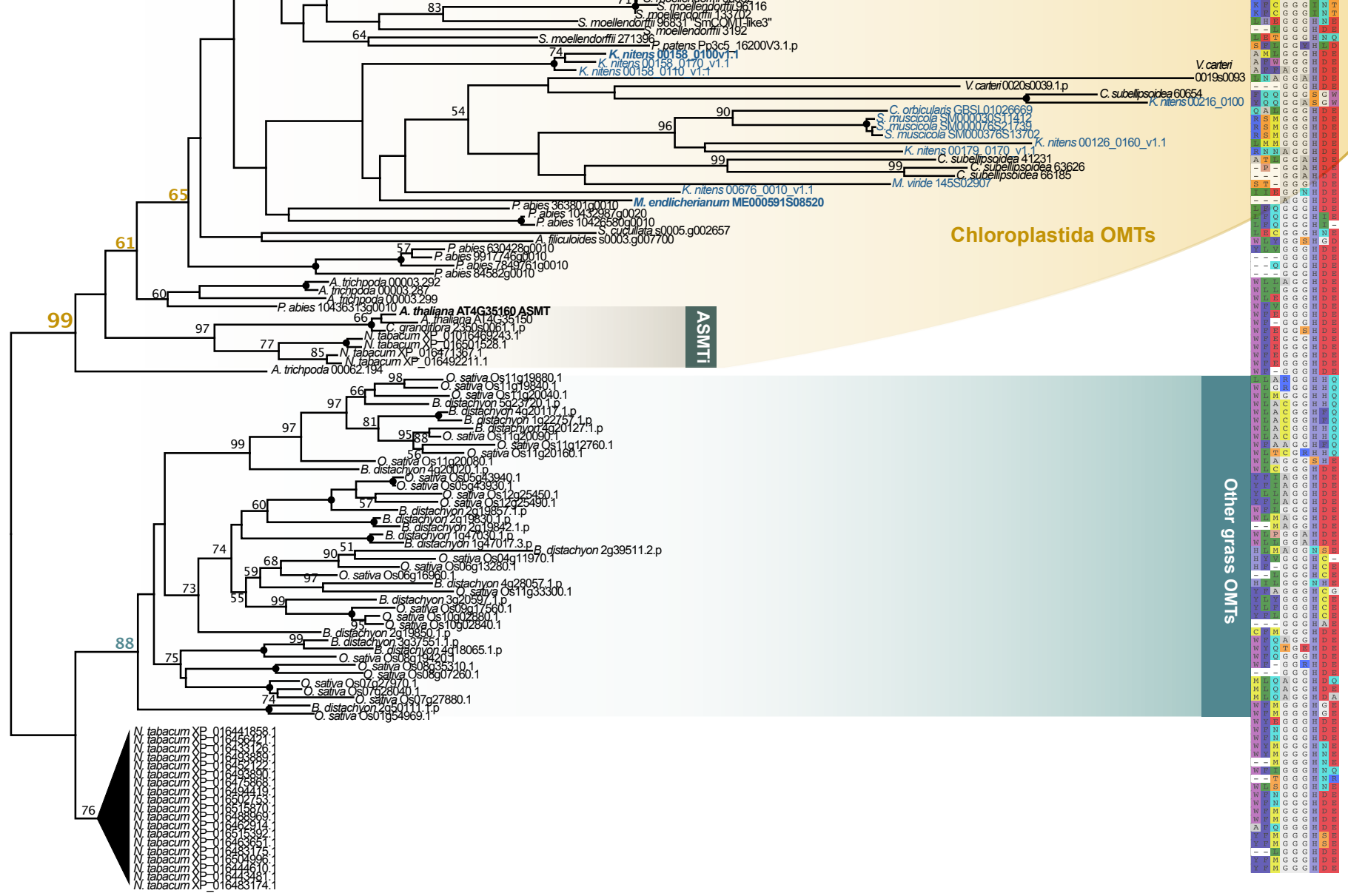




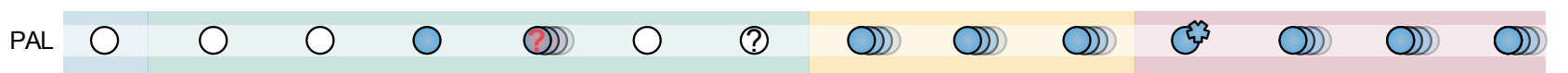
$\begin{array}{llllllllllllll}\mathrm{CH} & \mathrm{O} & \mathrm{O} & \mathrm{O} & \mathrm{O} & \mathrm{O} & \mathrm{O} & \mathrm{O} & 0 & 0 & 0 & 0 & 0 & 0\end{array}$

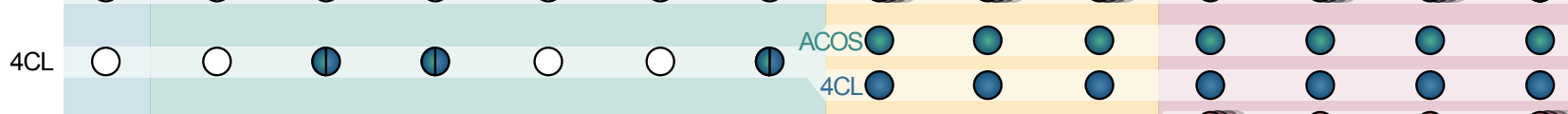

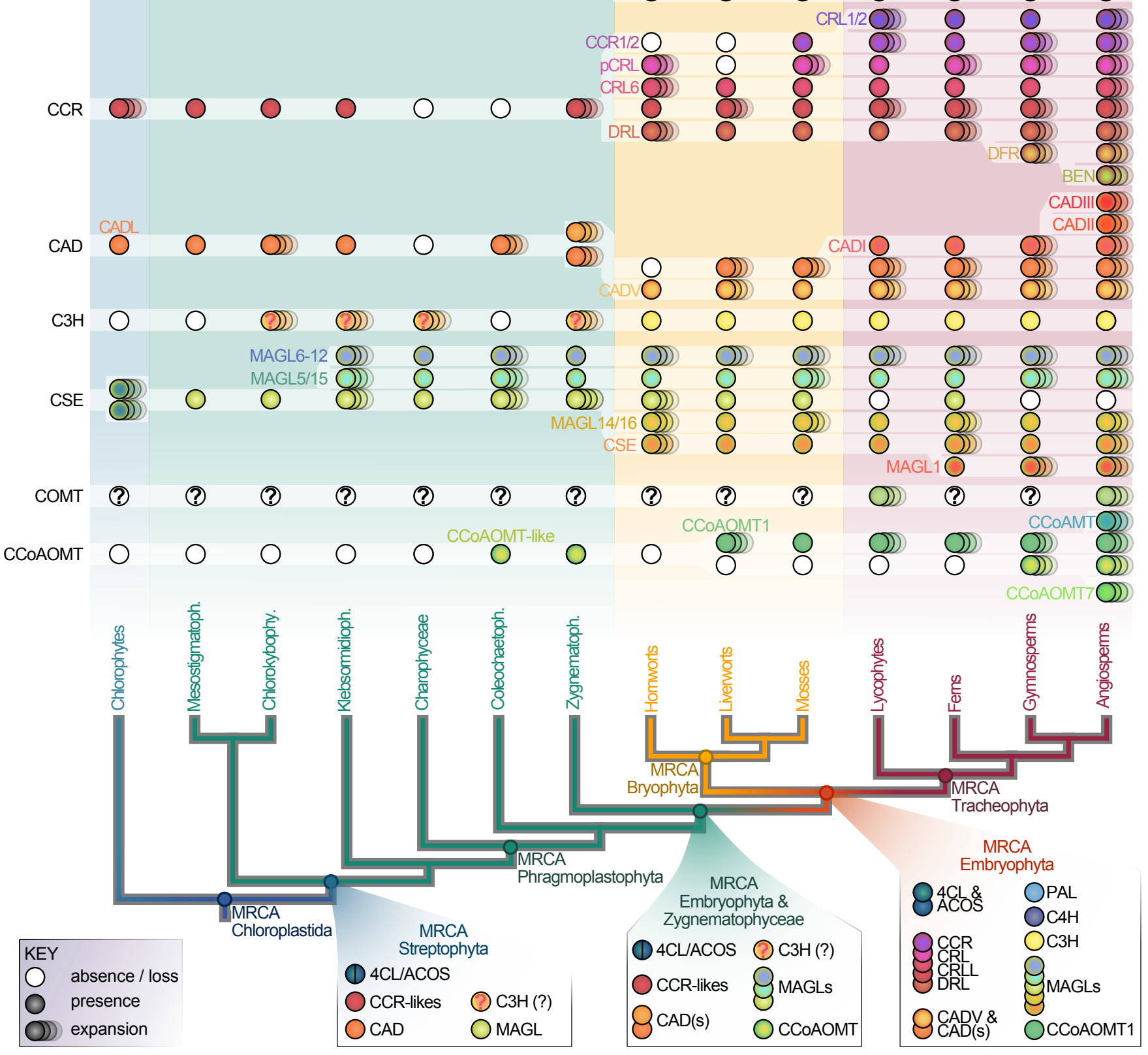

